\title{
Prinzipien der Osteosynthese im Bereich des Gesichtsschädels
}

Frank Wilde, Alexander Schramm

\author{
Während sich die Grundprinzipien der Osteosyntheseverfahren bei der Gesichts- \\ schädelrekonstruktion in den letzten Jahren wenig verändert haben, fand durch \\ Weiterentwicklung der Osteosynthesesysteme und Instrumente sowie durch die \\ Implementierung der computerassistierten Chirurgie einschließlich der Verwendung \\ anatomisch präformierter oder patientenspezifischer Implantate eine entschei- \\ dende Weiterentwicklung statt.
}

\section{Einleitung}

Die Platten-Schrauben-Osteosynthese zur Versorgung von Frakturen oder Rekonstruktion von Defekten hat sich seit Jahrzehnten im Mund-Kiefer-Gesichts-Bereich etabliert. Hierdurch wurde es möglich, den knöchernen Gesichtsschädel anatomisch dreidimensional zu rekonstruieren und auf eine längere starre mandibulomaxilläre Fixation von Ober- und Unterkiefer zu verzichten. Die stabile Fixierung mittels Osteosynthese folgt auch im Bereich des Gesichtsschädels den 4 Prinzipien der AO Guidelines zur adäquaten Behandlung von Frakturen:

1. anatomische Knochenreposition,

2. stabile Fixierung der Fragmente,

3. Erhalt der Durchblutung und

4. Sicherstellung einer frühen Funktion [1].

Im Laufe der Weiterentwicklung der Osteosynthese im Mund-Kiefer-Gesichts-Bereich wurden immer neue Systeme je nach Indikationsstellung entwickelt. Während noch Anfang dieses Jahrtausends die Platten-SchraubenSysteme hinsichtlich Schraubendurchmesser und Plattenstärke sowie hinsichtlich Schraubenprinzip - winkelstabil versus nicht winkelstabil - jeweils geschlossene Systeme darstellten, erlauben heutzutage moderne Osteosynthesesysteme eine multimodale Anwendung eines einzelnen Systems. Unterschieden wird in der Regel nur noch zwischen dem Einsatz im Ober- oder Unterkiefer ( $\vee$ Abb. 1 a und b). Zusätzlich unterscheidet man im Unterkiefer noch zwischen Traumatologie- und Rekonstruktionssystemen, wobei bei den Rekonstruktionssystemen im Vergleich zu den Traumasystemen prinzipiell nur die Plattenstärken und die Schraubendurchmesser nochmals zunehmen ( $\bullet$ Abb. 1 c).

Innerhalb eines Subsystems ist es jeweils uneingeschränkt möglich, alle unterschiedlichen Plattenstärken mit allen unterschiedlichen Schrauben zu verwenden. Neben dem Standardschraubendurchmesser stehen zusätzlich Schrauben mit einem geringfügig vergrößerten Durchmesser als sogenannte Emergency- bzw. RescueSchrauben zur Verfügung, falls es zu einem Durchdrehen der Schraube mit Standarddurchmesser kommt. Bei den Systemen für den Einsatz im Unterkiefer ist es zusätzlich möglich, sowohl winkelstabile als auch nicht winkelstabile Schrauben bei ein und derselben Platte einzusetzen und miteinander zu kombinieren. Das gleiche gilt auch für die zwar weiter vorhandenen speziellen Kompressionsplatten, die jedoch bei der modernen Osteosynthese im Unterkiefer inzwischen eine eher untergeordnete Rolle spielen und mehr und mehr durch Adaptationsplatten ersetzt wurden.

Durch diese multimodale Anwendung der modernen Osteosynthesesysteme ist es möglich, sowohl im Unter- als auch im Oberkiefer die Plattenstärken individuell an die Indikation hinsichtlich Stabilität und Funktionalität anzupassen, ohne auf unterschiedliche Osteosynthesesysteme zurückgreifen zu müssen.

\section{Merke}

Bei modernen Osteosynthesesystemen können alle unterschiedlichen Platten mit allen unterschiedlichen Schrauben kombiniert werden.

\section{Prinzipien der Osteosynthese im Unterkiefer}

Bei der Osteosynthese im Bereich des Unterkiefers spielt neben der Anatomie die Biomechanik des Unterkiefers während der Mastikation weiter die entscheidende Rolle bei den Überlegungen hinsichtlich der optimalen Positionierung von Osteosyntheseplatten. Bei der Einleitung von 

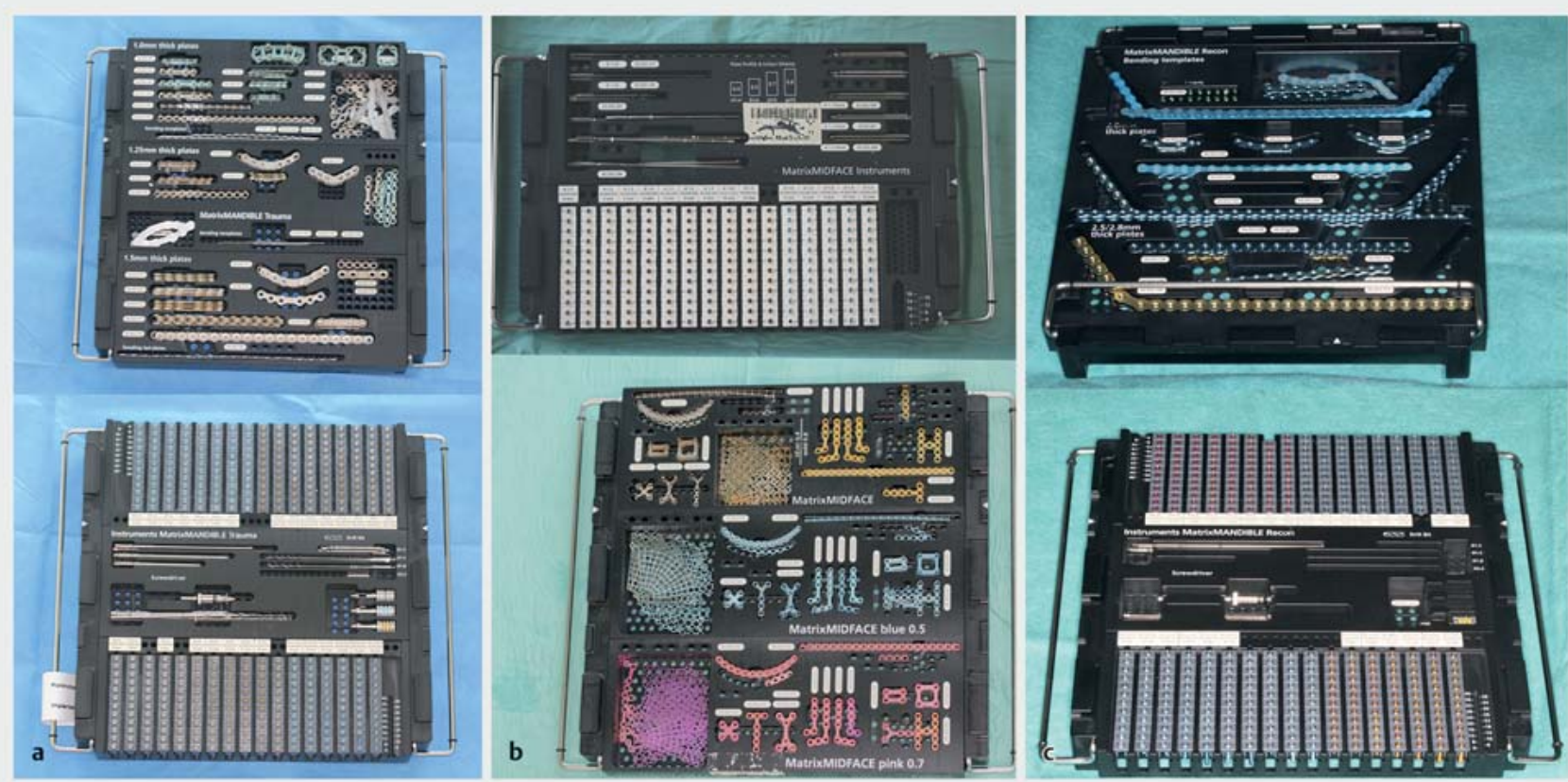

- Abb. 1 a Modernes Osteosynthesesystem Matrix Mandible Trauma (DePuy/Synthes, Schweiz). Alle Platten können mit allen unterschiedlichen Schrauben kombiniert werden. b Modernes Osteosynthesesystem Matrix Midface (DePuy/Synthes, Schweiz). Alle Platten können mit allen unterschiedlichen Schrauben kombiniert werden. c Modernes Osteosynthesesystem Matrix Mandible Recon (DePuy/Synthes,Schweiz). Alle Platten können mit allen unterschiedlichen Schrauben kombiniert werden.

Kräften auf den Unterkiefer durch Abbeißen und Kauen, kommt es zu verschiedenen Zonen von Kompression und Zugspannung. Diese sind abhängig vom Ort der Kräfteapplikation und unterscheiden sich danach, ob mit den Seitenzähnen Speisen zerkleinert werden oder ob mit den Frontzähnen abgebissen wird. Durch die Hufeisenform des Unterkiefers kommt es des Weiteren, über die einwirkenden Kau- und Muskelkräfte, zu einer dreidimensionalen Deformierung des Unterkiefers. Vereinfacht betrachtet resultiert beim Zusammenbiss im Bereich des Alveolarfortsatzes eine Zugzone, während sich im Bereich des basalen Unterkieferrandes eine Kompressionszone zeigt. Zwischen der Zug- und Kompressionszone liegt die sogenannte neutrale Zone, in der sich in der Theorie die beiden gegensätzlichen Kräfte gegenseitig aufheben ( $\bullet$ Abb. 2). Bezogen auf dieses Modell ist es vom biomechanischen Aspekt vorteilhaft, die Osteosynthese im Bereich der Zugzone oder der Neutralzone, also im Bereich des zahntragenden Anteils des Unterkiefers zu platzieren, um einem Auseinanderweichen der Frakturfragmente entgegenzuwirken. Anatomisch stellt sich die krestale Zugzone jedoch durch das Vorhandensein der Zähne, einer dünnen Kortikalis und einer dünnen darüberliegenden Schleimhaut als kompliziert dar. Somit bietet sich eine Platzierung der Osteosyntheseplatten im Bereich der Neutralzone an. Diese wurde auch als ideale Osteosyntheselinie definiert ( $\bullet$ Abb. 3). Diese korreliert im Unterkieferkorpus jedoch i.d.R. mit der Ebene auf
Höhe des N. mandibularis inferior, sodass hier nur eine monokortikale Verankerung von Schrauben möglich ist. Der Unterrand des Unterkiefers stellt vom biomechanischen Aspekt (Kompressionszone) her eigentlich eine eher weniger geeignete Lokalisation für die Positionierung von Osteosynthesematerial dar. Doch durch die dort vorhandene dicke Kortikalis und den sicheren Weichteilmantel bietet sich der basale Unterkieferrand dennoch als Lokalisationsort zur Befestigung von Osteosynthesematerial an. Insbesondere in der Kombination mit einer monokortikalen Osteosynthese im Bereich der Neutralzone [2].

Um einerseits den biomechanischen Anforderungen und andererseits den anatomischen Gegebenheiten bei der osteosynthetischen Versorgung des Unterkiefers Rechnung zu tragen, unterscheidet man heutzutage zwischen 2 Grundprinzipien der Fixierung:

1. Load-sharing-Osteosynthese

2. Load-bearing-Osteosynthese [2] ( $\mathbf{A} \mathbf{b b}$. 4a und b).

\section{Load-sharing-Osteosynthese}

Bei der Load-sharing-Osteosynthese stellt die exakte anatomische Reposition der Fraktur den zentralen Aspekt des Prinzips dar. Hierdurch wird es möglich, dass die auftretenden funktionellen Kräfte während der Unterkieferbewegung über die Fraktur hinweg durch den Knochen aufgenommen bzw. weitergeleitet werden, während das 


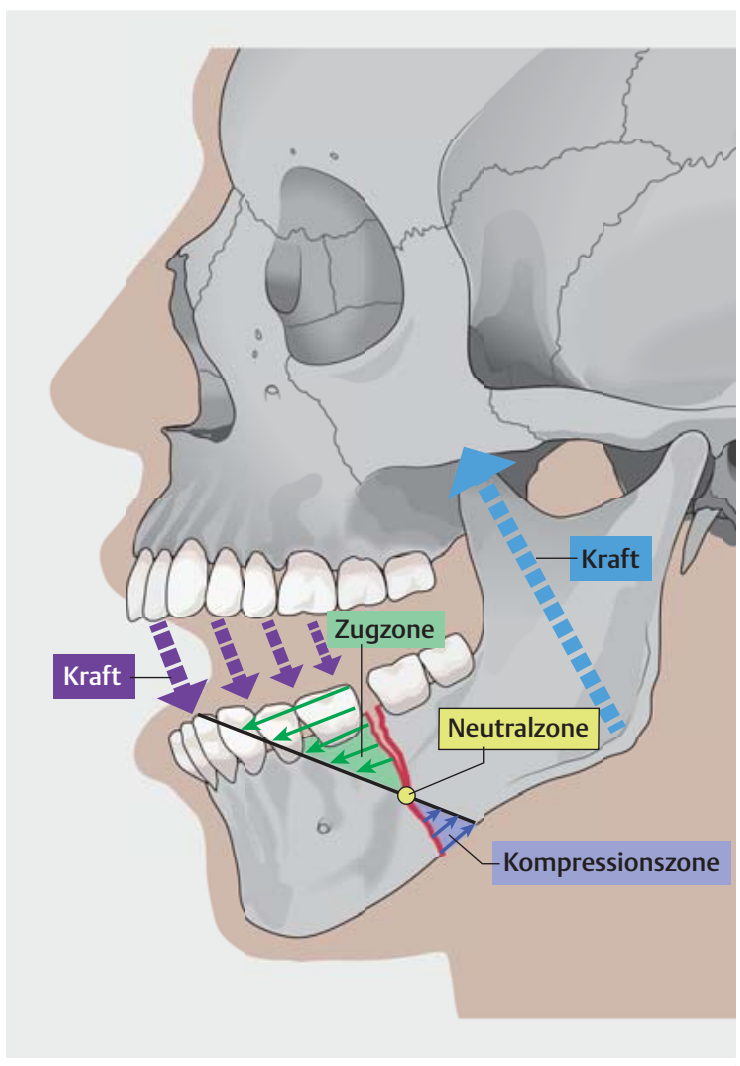

- Abb. 2 Biomechanik des Unterkiefers bei der Mastikation. Im Bereich des Alveolarfortsatzes entsteht eine Zugzone, im Bereich des basalen Unterkieferrandes eine Kompressionszone. Zwischen der Zug- und Kompressionszone liegt die sogenannte neutrale Zone, in der sich in der Theorie die beiden gegensätzlichen Kräfte gegenseitig aufheben.

Osteosynthesematerial die Fragmente nur zueinander fixiert und stabilisiert. Das Osteosynthesematerial muss demnach nur einen geringen Anteil der auftretenden Kräfte auffangen, was wiederum dazu führt, dass dünne Osteosyntheseplatten der Profilstärken 1,0-1,5 mm Verwendung finden können, welche i.d. R. monokortikal mit Schraubendurchmessern von ca. 2,0 mm aufgeschraubt werden ( $\bullet$ Abb. 1 a). Das Prinzip der Load-sharing-Osteosynthese kommt somit bei der Miniplattenosteosynthese im Bereich des Unterkiefers in zahntragenden Anteilen, im Kieferwinkel oder im Bereich des Gelenkfortsatzes zur Anwendung. Die Miniplatten sollten dabei so lang gewählt werden, dass jeweils mindestens 2 Schrauben je instabiler Frakturseite sicher monokortikal verankert werden können, um eine Rotationsstabilität zu gewährleisten.

Der operative Zugang ist i. d. R. ein intraoraler. Durch den Einsatz eines $90^{\circ}$ abgewinkelten Bohrers und Schraubendrehers ist dies auch in der überwiegenden Anzahl der Fälle, selbst bei Gelenkfortsatzfrakturen, möglich.

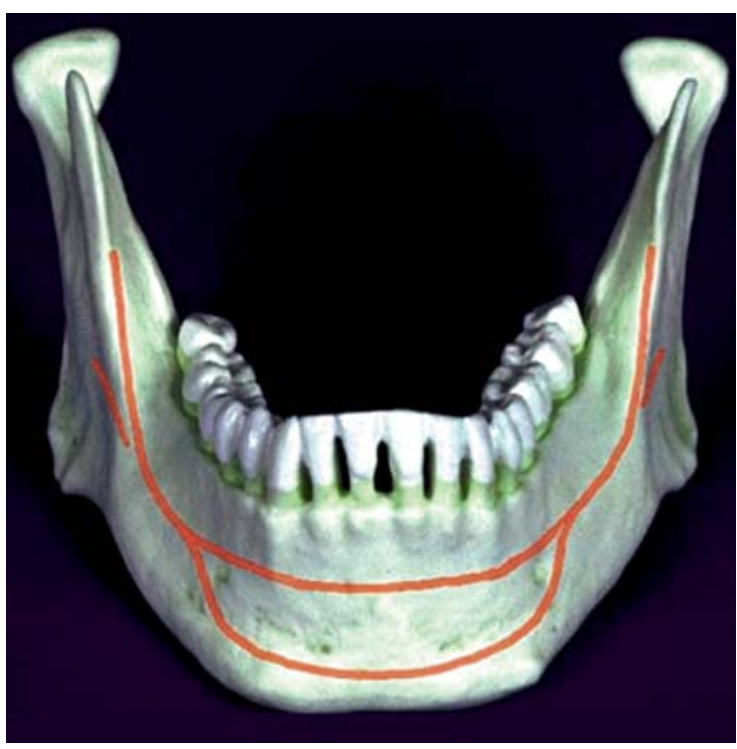

- Abb. 3 Ideale Osteosyntheselinie im Unterkiefer.

\section{Load-bearing-Osteosynthese}

Bei der sogenannten Load-bearing-Osteosynthese wird die gesamte einwirkende Kraft durch das Osteosynthesematerial getragen. Hierbei wird dickeres Osteosynthesematerial der Profilstärken 2,0-3,0 mm mit bikortikaler Schraubenverankerung eingesetzt. Die eingesetzten Schraubendurchmesser bewegen sich in Abhängigkeit vom Hersteller zwischen 2,3-3,0 mm ( $\bullet$ Abb. 1 c). Es empfiehlt sich, je Fraktur- bzw. Defektseite mindestens 3, besser 4-5 Schrauben zu platzieren.

Die Indikationen für eine Load-bearing-Osteosynthese sind komplexe mehrfragmentäre Frakturen, Defektfrakturen, Frakturen des atrophierten Unterkiefers, pathologische Frakturen, Komplikationen nach Frakturen mit Pseudoarthrose und Infektionen sowie Kontinuitätsdefekte im Rahmen der Unterkieferrekonstruktion.

Mit Ausnahme vielleicht im Bereich des anterioren Unterkiefers wird bei einer Load-bearing-Osteosynthese häufig ein extraoraler Zugang zum Unterkiefer notwendig.

\footnotetext{
Merke

Je dicker der Knochen, desto dünner kann die Platte gewählt werden.

Je dünner der Knochen, desto dicker sollte die Platte gewählt werden.
}

Die neuesten Entwicklungen gehen in Richtung anatomisch präformierte Osteosyntheseplatten. Diese sollen das Konturieren der Platten erleichtern, die Plattenfrakturgefahr mindern und Operationszeiten reduzieren ( Abb. 5). 


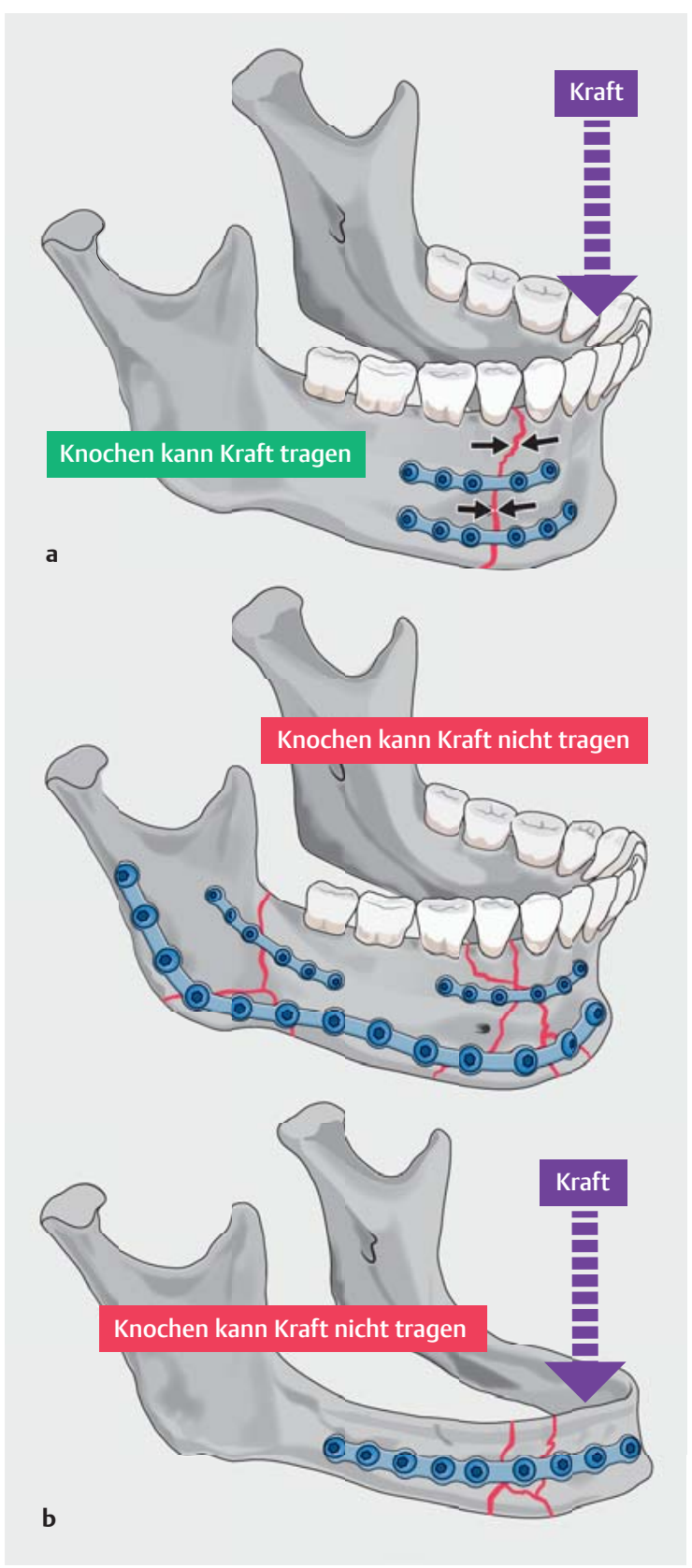

- Abb. 4 Grundprinzipien der Osteosynthese im Unterkiefer. a Load-sharing-Osteosynthese, b Load-bearingOsteosynthese.

\section{Anwendungsbeispiele der Osteosynthese im Unterkiefer}

\section{Paramediane/mediane Unterkieferfraktur}

Bei diesem sehr häufigen Frakturmuster stellt die Miniplattenosteosynthese mit 2 Miniplatten in der Regel die osteosynthetische Versorgung der Wahl dar. Dabei kommen nach anatomischer Reposition und Sicherstellung der Okklusion über eine mandibulomaxilläre Fixation (z.B. mittels MMF-Schrauben) 2 Osteosyntheseplatten zum Einsatz. Dabei wird eine Osteosyntheseplatte knapp unterhalb der Zahnwurzeln in der neutralen Zone platziert. Eine weitere Miniosteosyntheseplatte kommt im Bereich des basalen Unterkieferrandes zu liegen ( Abb. 6a). Es empfiehlt sich, die Platten leicht zu überbiegen, um dem Phänomen des lingualen Aufbiegens entgegenzuwirken.

Bei streng medianen Unterkieferfrakturen kann auch eine alleinige Zugschraubenosteosynthese zum Einsatz kommen. Dies gestaltet sich jedoch nach Autorenmeinung häufig komplizierter als die Plattenosteosynthese. Weiter empfiehlt es sich bei streng medianen Unterkieferfrakturen, neben dem Überbiegen der Platten, die basale Platte etwas dicker zu wählen als die krestale, insbesondere wenn gleichzeitig eine beidseitige Gelenkfortsatzfraktur vorliegt ( Abb. 6b).

\section{PRAXISTIPP}

Neben dem Überbiegen der Platte kann während der Osteosynthese durch temporäres Einsetzen einer großen spitzen Repositionsklemme/-zange im Bereich der krestalen Molarenregion eine gut dosierbare, posteriore Kompression ausgeübt werden. Hierdurch kann einer lingualen Spaltbildung und einer Verbreiterung des Unterkiefers suffizient entgegengewirkt werden. Dies trifft insbesondere auf mediane und paramediane Frakturen mit Ausgleichsfrakturen im Bereich der Gelenkfortsätze oder der Kieferwinkel zu.

\section{Kieferwinkelfrakturen}

Obwohl bei einfachen Kieferwinkelfrakturen nach den Untersuchungen von Champy eine einzelne Platte im Bereich der Zugzone, auf der Linea obliqua, ausreichend ist [2,3], empfiehlt es sich nach Autorenmeinung, auch bei Kieferwinkelfrakturen 2 Osteosyntheseplatten einzusetzen. Dabei kommt die eine Osteosyntheseplatte ebenfalls im Bereich der Zugzone entweder lateral oder direkt auf der Linea obliqua zu liegen, während die zweite Miniosteosyntheseplatte im Bereich des basalen Unterkieferrandes aufgeschraubt wird ( $\bullet$ Abb. $7 \mathbf{a}$ ). Durch diese Fixierung mit 2 Miniplatten kann eine basale Spaltbildung im Bereich der eigentlichen Druckzone, wie es sich klinisch häufig bei der Versorgung von Kieferwinkelfrakturen mit nur einer Platte auf der Linea obliqua zeigt, in der Regel verhindert werden ( $\bullet$ Abb. $7 \mathbf{b}$ ). Durch den Einsatz des $90^{\circ}$ abgewinkelten Schraubendrehers ist die Fixation beider Platten durch einen ausschließlich intraoralen Zugang möglich. Alternativ bestünde für die kaudale Platte die Fixation mittels eines transbukkalen Zugangs. Dies wird von den Autoren jedoch nur im Einzelfall empfohlen.

Im Bruchspalt liegende Weisheitszähne sollten, wenn sie kein Repositionshindernis darstellen oder nicht zerstört 

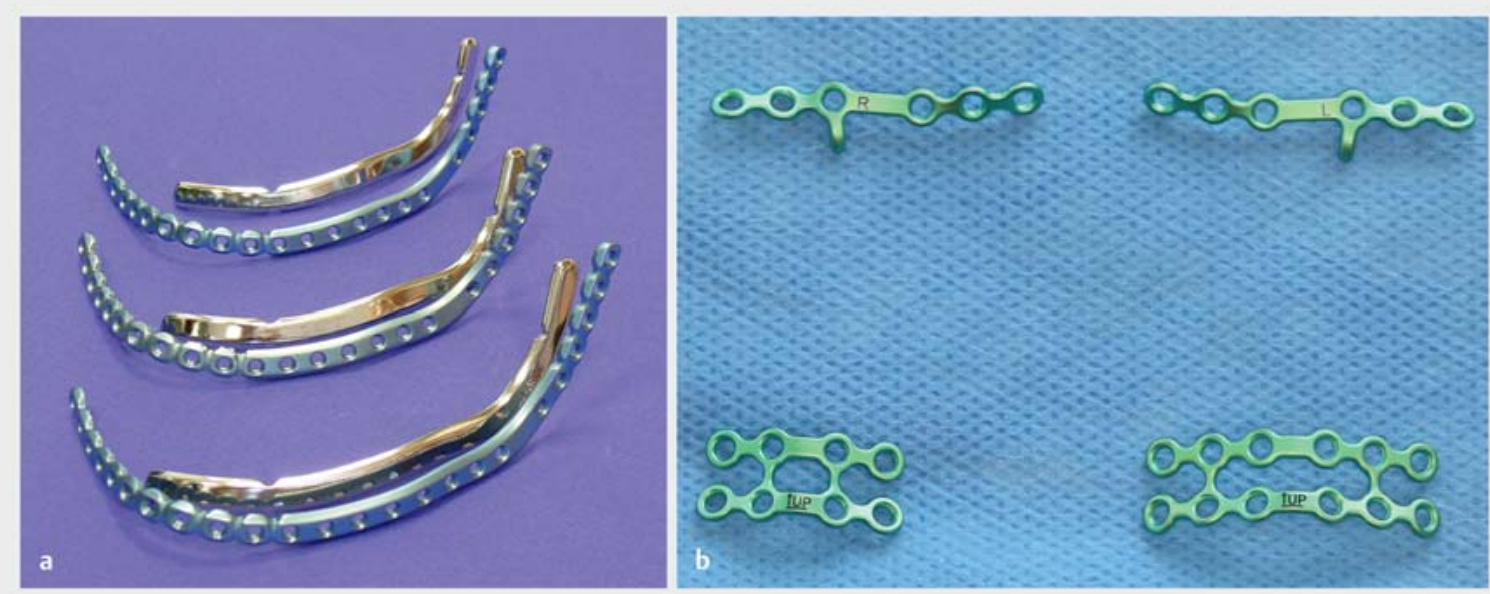

- Abb. 5 Anatomisch präformierte Platten für den Unterkiefer. a Anatomisch präformierte Unterkieferrekonstruktionsplatten. Diese liegen für rechts und links jeweils in 3 Größen vor (DePuy/Synthes, Schweiz). b Anatomisch präformierte Miniplatten für den Kieferwinkel im Bereich der Linea obliqua (oben) und den medianen Unterkiefer (unten) (KLS-Martin, Deutschland).
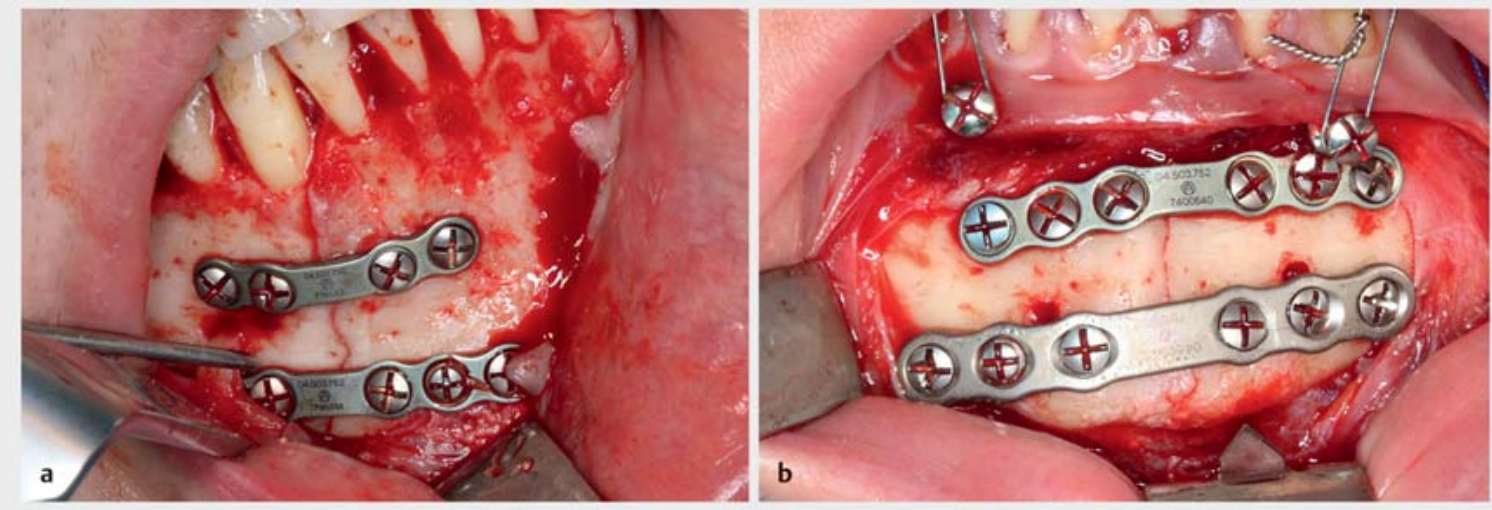

- Abb. 6 a Typische osteosynthetische Versorgung einer paramedianen Unterkieferfraktur mit 2 Miniplatten. b Osteosynthetische Versorgung einer medianen Unterkieferfraktur in Kombination mit beidseitiger Gelenkfortsatzfraktur mit 2 Osteosyntheseplatten. Die basale Platte wurde aus Stabilitätsgründen in diesem Fall etwas dicker gewählt.

sind, belassen werden. Denn durch eine Entfernung verliert man unweigerlich Stabilität nach dem Prinzip des Load-sharing und schafft sich im Einzelfall sogar eine Defektfraktur, die eine Load-bearing-Osteosynthese erforderlich machen könnte. Die Entscheidung, ob Zähne aus dem Frakturspalt intraoperativ entfernt werden müssen bzw. sollten, ist jedoch jeweils individuell zu entscheiden.

\section{Unterkieferkorpusfraktur}

Aus Stabilitätsgründen empfiehlt sich auch hier im Regelfall der Einsatz von 2 Miniosteosyntheseplatten, wovon eine wiederum auf Höhe der Neutralzone knapp unterhalb der Wurzelspitzen und oberhalb des N. mandibularis zu liegen kommt. Die 2. Platte wird im Bereich des basalen Unterkieferrandes unterhalb des Nerven befestigt. Auch hier ist der Einsatz eines Winkelschraubendrehers, insbesondere im Bereich der basalen Platte, häufig hilfreich.

Bei Schrägfrakturen des Corpus mandibulae empfiehlt sich dagegen auch der Einsatz einer Zugschraubenosteosynthese, um die schräg zueinander verlaufenden Frakturhälften aufeinander zu pressen ( $\bullet \mathbf{A b b} \mathbf{8} \mathbf{8}$ und $\mathbf{b}$ ). Dabei ist jedoch von entscheidender Bedeutung, dass bei der Platzierung der bikortikalen Zugschrauben der N. mandibularis sowie die Zahnwurzeln nicht beschädigt werden. Dies kann im Einzelfall schwierig werden, sodass sich in einem solchen Fall eine Zugschraubenosteosynthese verbietet. Der Vorteil der Zugschraubenosteosynthese ist der hohe Grad der Stabilität bei idealer Krafteinleitung in den Knochen. Im Einzelfall ist auch eine Kombination aus Miniplattenosteosynthese und Zugschraubenosteosynthese sinnvoll. 

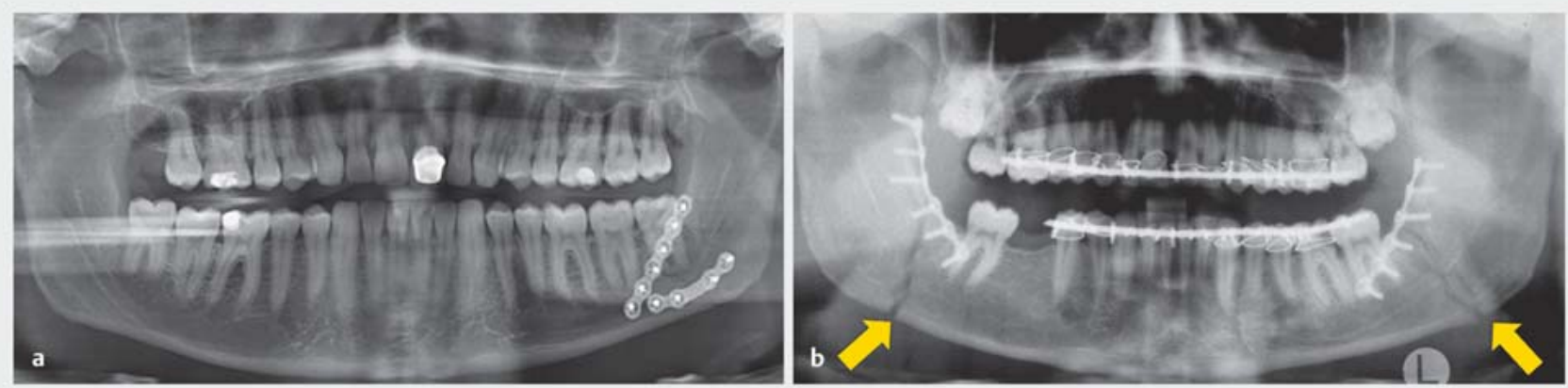

- Abb. 7 a Osteosynthetische Versorgung einer Kieferwinkelfraktur mit 2 Miniplatten. Eine ist auf Höhe der Linea obliqua krestal positioniert. Eine weitere am basalen Unterkieferrand. Hierdurch wird Aufbiegen in diesem Bereich verhindert. Der Weisheitszahn im Bruchspalt wurde bewusst belassen. b Typisches Aufbiegen des basalen Kieferwinkels bei der Versorgung einer Kieferwinkelfraktur mit nur einer Platte krestal im Bereich der Linea obliqua.
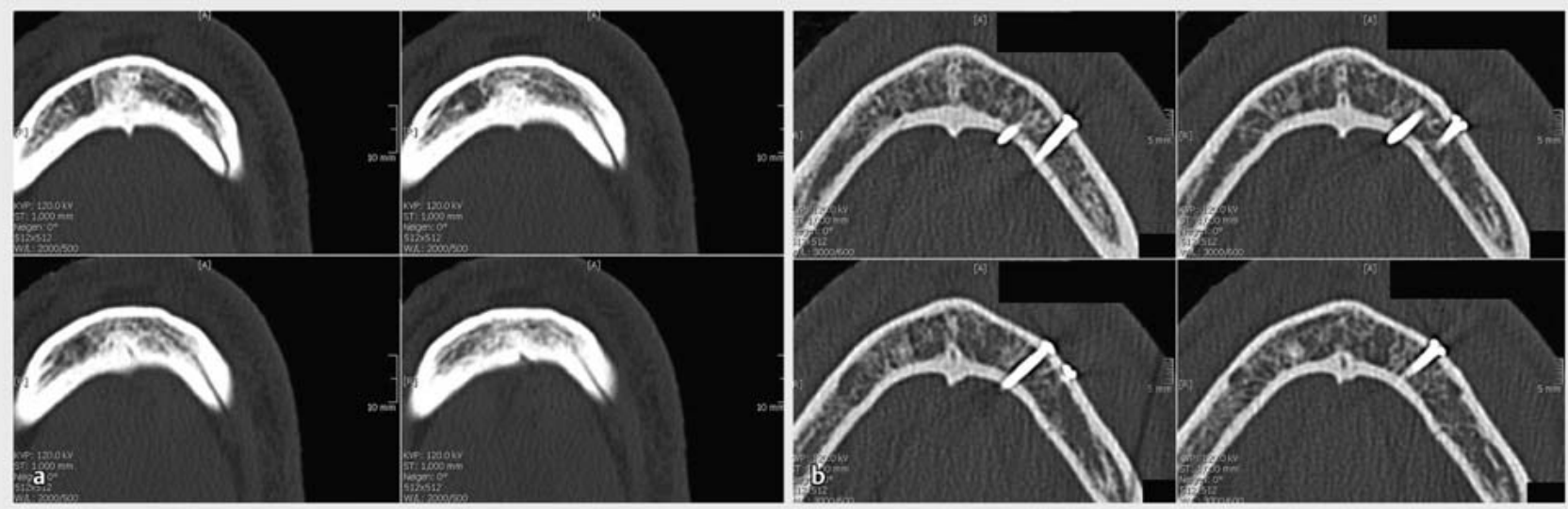

- Abb. 8 a Unterkieferschrägfraktur präoperativ. b Mit 3 Zugschrauben versorgte Unterkieferschrägfraktur postoperativ.

\section{PRAXISTIPP}

Bei der Unterkiefer-Miniplattenosteosynthese empfiehlt es sich nach anatomischer Reposition, primär die krestale Platte mit 2 exzentrisch gebohrten, frakturspaltnahen Schrauben zu fixieren, um in der Zugzone eine Kompression im Bereich des Frakturspaltes auszuüben. Vor der rotationsstabilen Fixierung der Platte mit den übrigen Schraubenlöchern sollte jedoch jetzt erst die basale 2. Osteosyntheseplatte fixiert werden. Hierdurch wird es möglich, durch die noch bestehende Rotationsmöglichkeit um die krestale Platte einer basalen Spaltentwicklung entgegenzuwirken, indem man z. B. mittels einer Repositionszange gezielt diesen Spalt schließt, bevor man die Osteosyntheseplatte aufschraubt. Auch hier empfiehlt es sich, die beiden ersten frakturnahen Löcher exzentrisch auf Kompression zu bohren. Erst dann sollten die beiden Platten mit weiteren Schrauben definitiv fixiert werden.

\section{Gelenkfortsatzfrakturen}

Bei dislozierten Gelenkfortsatzfrakturen empfiehlt sich ebenfalls die Miniplattenosteosynthese. In Abhängigkeit vom Platzangebot sollten, wenn möglich, auch hier 2 Osteosyntheseplatten aus Stabilitätsgründen verwendet werden. Dabei sollte eine im Bereich des Hinterrandes des aufsteigenden Astes und eine weitere im Bereich der Incisura mandibulae zu liegen kommen ( $\bullet$ Abb.9). Bei der Verwendung einer singulären Platte sollte diese so dimensioniert sein, dass diese die auftretenden Kräfte komplett übernehmen kann. Auch werden von Herstellern verschiedene Spezial-3-D-Platten angeboten, um hier dem Problem von Plattenfrakturen entgegenzuwirken.

Bezüglich des operativen Zugangs gibt es grundsätzlich 2 Möglichkeiten. Die eine stellt den extraoralen Zugang in der Regel präaurikulär oder retromandibulär dar. Wobei jedoch auch andere Zugangswege wie submandibulär oder retroaurikulär möglich sind [4]. Der Vorteil des extraoralen Zugangs ist der direkte Zugang und die verbesserte Visualisierung der Fraktur für die Reposition und 
Osteosynthese. Mit dem extraoralen Zugang einhergehende Nachteile sind einerseits die extraorale Narbe sowie andererseits die Gefahr der Verletzung des N. facialis [2].

Als alternativer Zugang zum Gelenkfortsatz bietet sich der in der Autorenklinik favorisierte intraorale Zugang an, der endoskopisch unterstützt zur Anwendung kommt ( Abb. 10). Der Zugang ähnelt dabei dem Zugang zu einer Unterkieferosteotomie im Rahmen einer sagittalen Spaltung des Unterkiefers, wobei hier die laterale Präparation weiter nach kranial erfolgen muss ( $\mathbf{A} \mathbf{A b} \mathbf{b} \mathbf{1 1})$. Des Weiteren sind aufgrund der limitierten Sicht Spezialinstrumente hilfreich. Hierzu zählen überlange Raspatorien und Repositionshäkchen ( $\mathbf{A} \mathbf{b b} \mathbf{b}$ 12) sowie eine lichtstarke Stirnleuchte. Zwingend erforderlich ist auch ein $90^{\circ}$ abgewinkelter Schraubendreher und Bohrer. Dieser sollte über eine Haltevorrichtung verfügen, die es ermöglicht, Platte mit Schraube zusammen einzubringen ( Abb. 13).

Neuste Entwicklungen von Osteosyntheseplatten gehen in Richtung anatomisch präformierter 3-D-Platten mit integrierter Repositionshilfe für den Gelenkfortsatz $(\triangleright$ Abb. 14).

\section{Unterkieferstückfrakturen}

Auch bei Unterkieferstückfrakturen mit 2 Frakturstellen, z. B. paramedian sowie im Kieferwinkel oder im Gelenkfortsatzbereich kontralateral, werden die gleichen bereits beschriebenen Prinzipien der Osteosynthese mit Reposition und stabiler Fixierung verfolgt, wie sie bei isolierten Frakturen gelten ( $\bullet$ Abb. 15).

\section{Komplexe Mehrfragmentfrakturen des Unterkiefers}

Bei komplexen Mehrfragmentfrakturen des Unterkiefers im Bereich des anterioren Unterkiefers, des Corpus man-

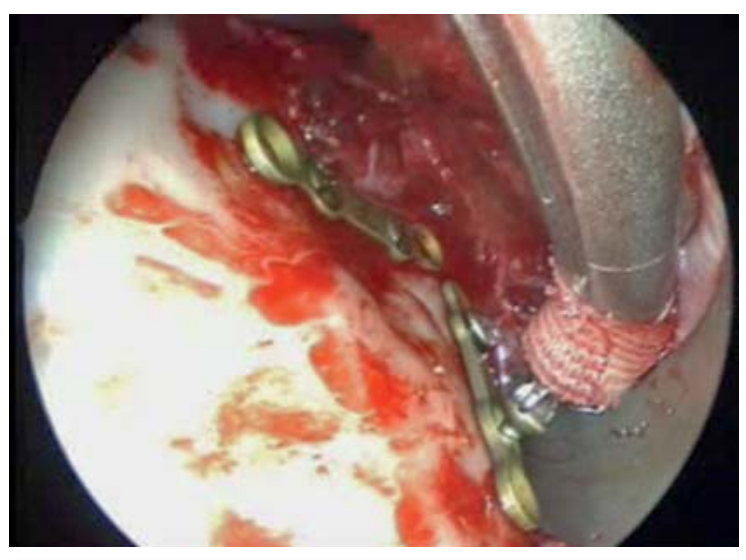

- Abb. 10 Endoskopischer Blick während der Osteosynthese einer Gelenkfortsatzfraktur über einen intraoralen Zugang.

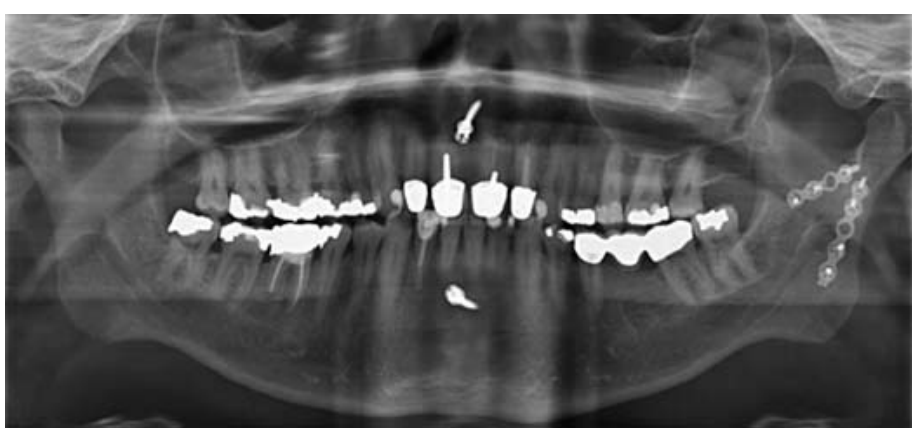

- Abb. 9 Mit 2 Miniplatten von intraoral versorgte linksseitige Gelenkfortsatzfraktur.

dibulae oder des Kieferwinkels, wird in der Vielzahl der Fälle wie bereits oben erwähnt, eine sogenannte Loadbearing-Osteosynthese mit dickeren Osteosyntheseplatten erforderlich. Auch eine Kombination aus Miniplatten, Zugschrauben und dicken Osteosyntheseplatten kommt hier je nach Frakturmuster zum Einsatz ( $\triangleright$ Abb. 16a). Dabei sollte mittels Miniplatten die Fraktur initial vereinfacht werden, um sie dann über dickeres Osteosynthesematerial zu stabilisieren.

Während im Bereich des anterioren Unterkiefers sowie des anterioren Corpus mandibulae dies meist noch mittels intraoralen Zugang bewerkstelligt werden kann, ist

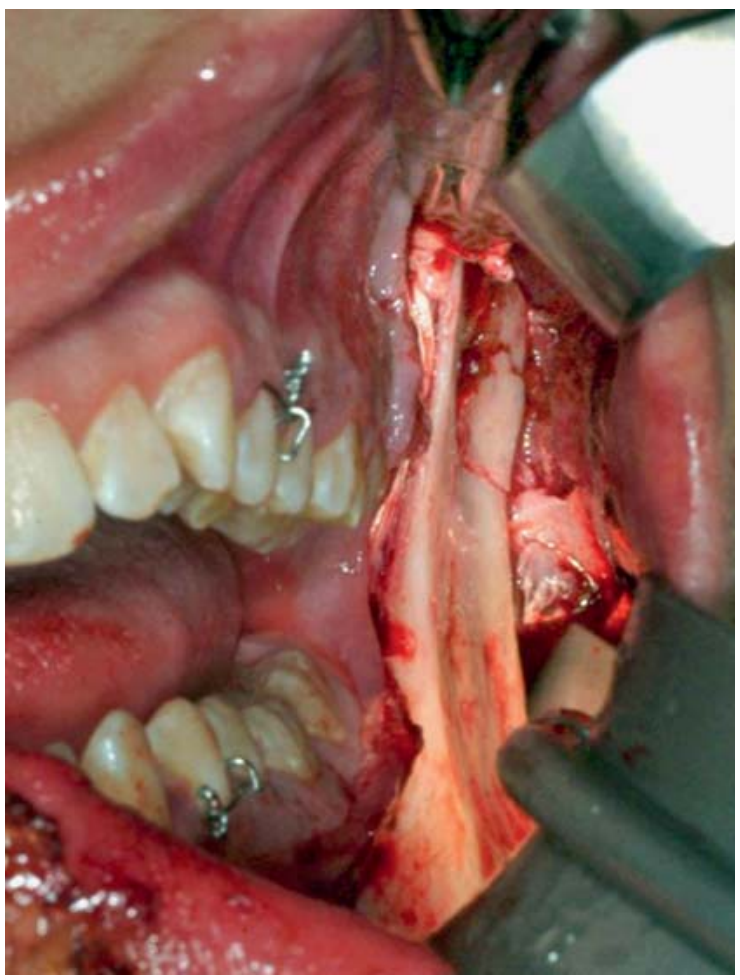

Abb. 11 Intraoraler Zugang zur Versorgung von Gelenkfortsatzfrakturen. 


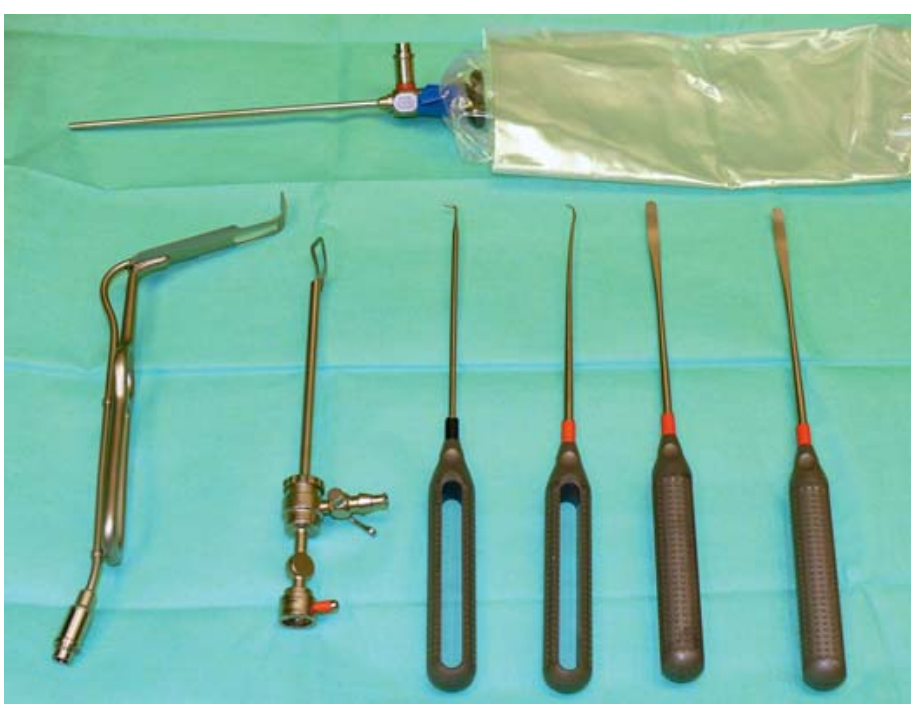

- Abb. 12 Überlange Spezialinstrumente, Endoskop und spezieller Lichtleiterhaken zur intraoralen Versorgung von Gelenkfortsatzfrakturen (Karl Storz, Deutschland).

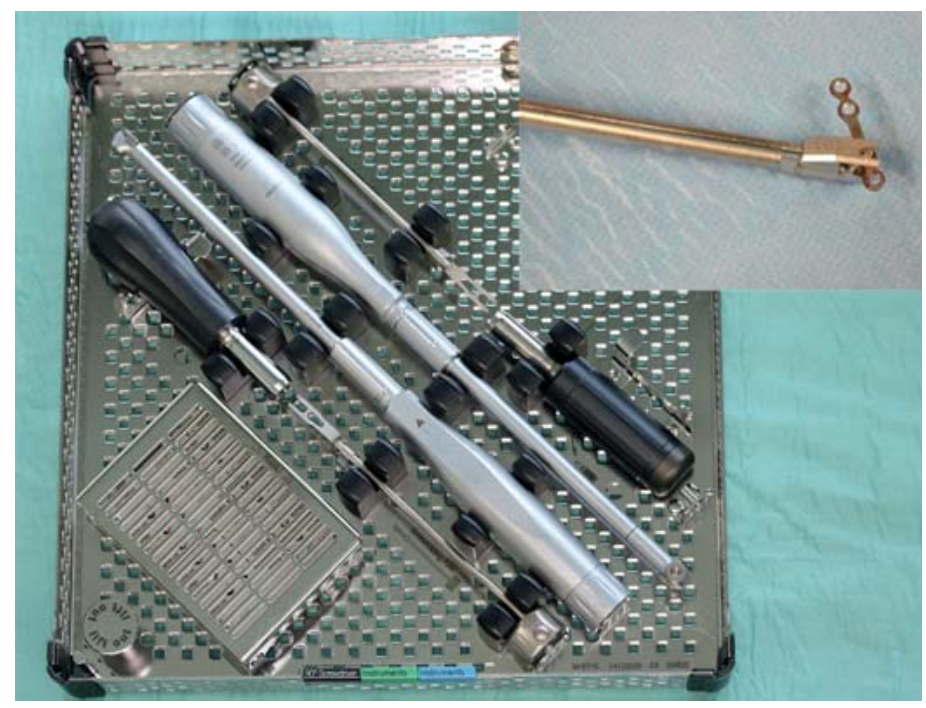

- Abb. $1390^{\circ}$ abgewinkelter Schraubendreher und Bohrer. Mittels Haltevorrichtung können Platte und Schraube in Kombination fixiert werden (MedArtis, Schweiz).

im Bereich des Kieferwinkels häufig ein extraoraler Zugang vorteilhaft. Dennoch ist es bei exakter Reposition und guter Compliance des Patienten auch möglich, solche Frakturen nur mittels Miniplatten durch einen intraoralen Zugang zu versorgen ( $\bullet$ Abb. $\mathbf{1 6 b}$ ).

\section{Frakturen im hoch atrophen Unterkiefer}

Frakturen im hoch atrophen Unterkiefer erfordern in der Regel eine Load-bearing-Osteosynthese mit dickem Osteosynthesematerial in Kombination mit winkelstabilen bikortikalen Schrauben. Ein extraoraler submandibulärer

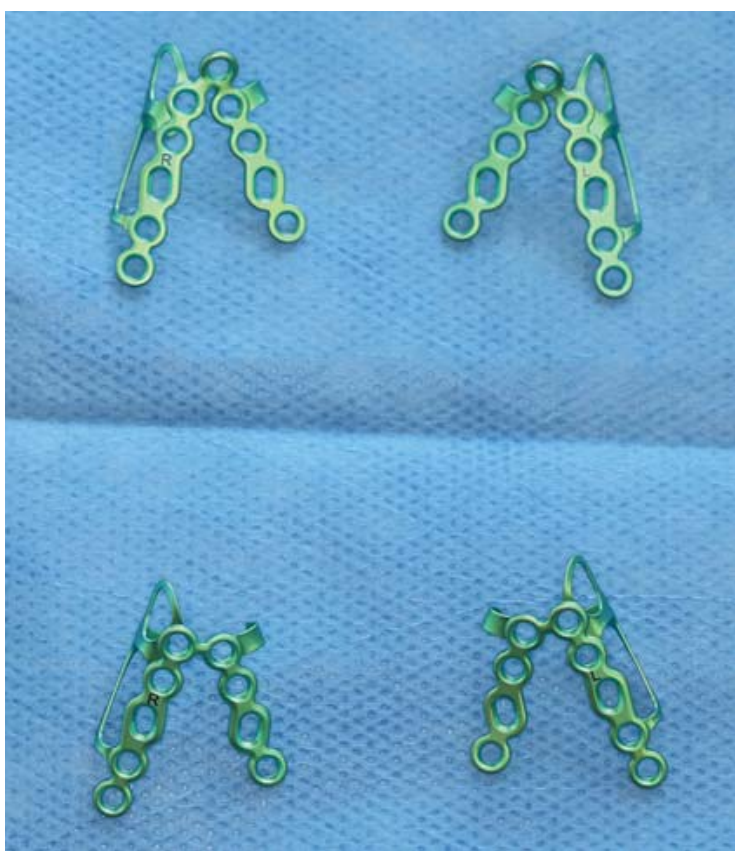

Abb. 14 Anatomisch präformierte 3-D-Platten mit integrierter Repositionshilfe für den Gelenkfortsatz (KLSMartin, Deutschland).

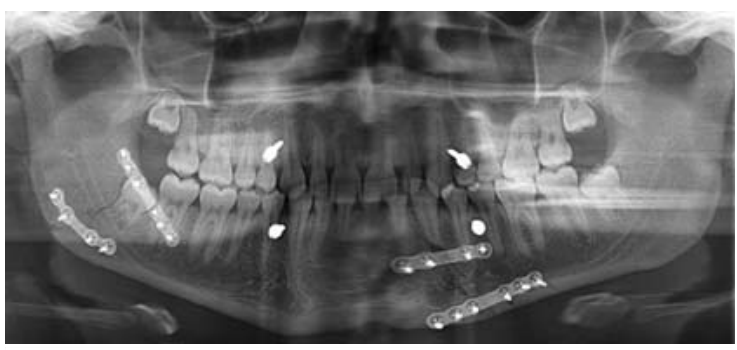

Abb. 15 Mittels Miniplatten versorgte Unterkieferstückfrakturen mit Paramedianfraktur links und Kieferwinkelfraktur rechts.

Zugang sollte präferiert werden. Dabei ist die Extension der Osteosyntheseplatte in der Regel vom aufsteigenden Unterkieferast bis zum Kinn und bei Stückfrakturen in beiden Unterkieferkörpern vom rechten bis zum linken Kieferwinkel notwendig ( $\boldsymbol{A}$ Abb. 17). Die bikortikalen Schrauben sollten hierbei im Bereich des Kieferwinkels und des aufsteigenden Unterkieferastes sowie im Bereich des anterioren Unterkiefers positioniert werden, da hier in der Regel weiter ausreichendes Knochenangebot zu finden ist. Eine weitere Möglichkeit besteht darin, die Platte von lingual zu positionieren [5]. Der Einsatz einer Miniplattenosteosynthese im hoch atrophen Unterkiefer ist in der Regel nicht suffizient und führt in den allermeisten Fällen zu einer Lockerung des Osteosynthesematerials, sodass ein solcher Versuch generell unterbleiben sollte. 

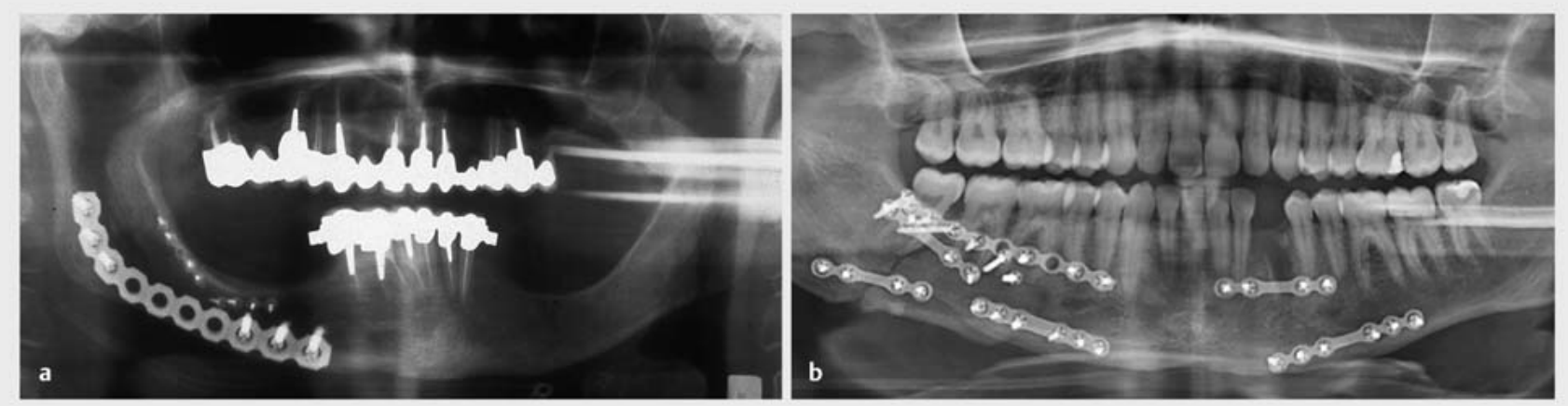

- Abb. 16 a Load-bearing-Osteosynthese einer rechtsseitigen Unterkieferstückfraktur im atrophen Unterkiefer. b Über intraoralen Zugang mittels Miniplatten und Zugschrauben versorgte komplexe Mehrfragmentfraktur des Unterkieferkorpus bei einem Patienten mit hoher Compliance.

\section{Unterkieferdefektfrakturen}

Bei Unterkieferdefektfrakturen z. B. aufgrund von Frakturen nach Weisheitszahnextraktionen oder nach infizierten Frakturen mit Sequestrierung von einzelnen Knochenanteilen ist ebenfalls eine Osteosynthese nach den Prinzipien des Load-bearings notwendig. Dabei sollten die Osteosyntheseplatten unter Verwendung von winkelstabilen Schrauben im Bereich des basalen Unterkieferrandes fixiert werden ( $\bullet$ Abb. 18).

In den meisten Fällen ist hierzu ebenfalls ein extraoraler Zugang sinnvoll. Dennoch kann in Einzelfällen auch durch einen intraoralen Zugang unter Verwendung eines $90^{\circ}$ Winkelbohrers und Drehers das Osteosynthesematerial eingebracht werden.

\section{Unterkieferkontinuitätsdefekte}

Bei der rein alloplastischen Rekonstruktion von Unterkieferkontinuitätsdefekten aufgrund einer Unterkieferteilresektion ist eine funktionsstabile Osteosynthese mit einer Rekonstruktionsplatte der Profilstärke 2,4-3,0 mm, die den Defekt weitstreckig überbrückt, obligat.

Hierzu werden seit einigen Jahren vermehrt patientenindividuelle CAD/CAM-Platten eingesetzt [6,7]. Da bei diesen Plattenfrakturen, wie sie bei Standardplatten regelhaft auftreten, eher selten, oder nicht zu erwarten sind, empfehlen die Autoren, wenn immer möglich solche Platten mit mindestens 5-6 bikortikalen Schrauben je Defektseite zu befestigen, da es möglicherweise durch die Rigidität solcher CAD/CAM-Platten unter Langzeitfunktion zum Versagen der Schrauben-Knochen-Verbindung kommen könnte ( $\bullet$ Abb. 19).

Bei einer knöchernen Rekonstruktion eines Unterkieferdefektes mit einem mikrovaskulären Knochentransplantat kommen, je nach Präferenz des Chirurgen, sowohl Miniplatten als auch Rekonstruktionsplatten erfolgreich zum Einsatz. Insbesondere für diesen Einsatzbereich sind

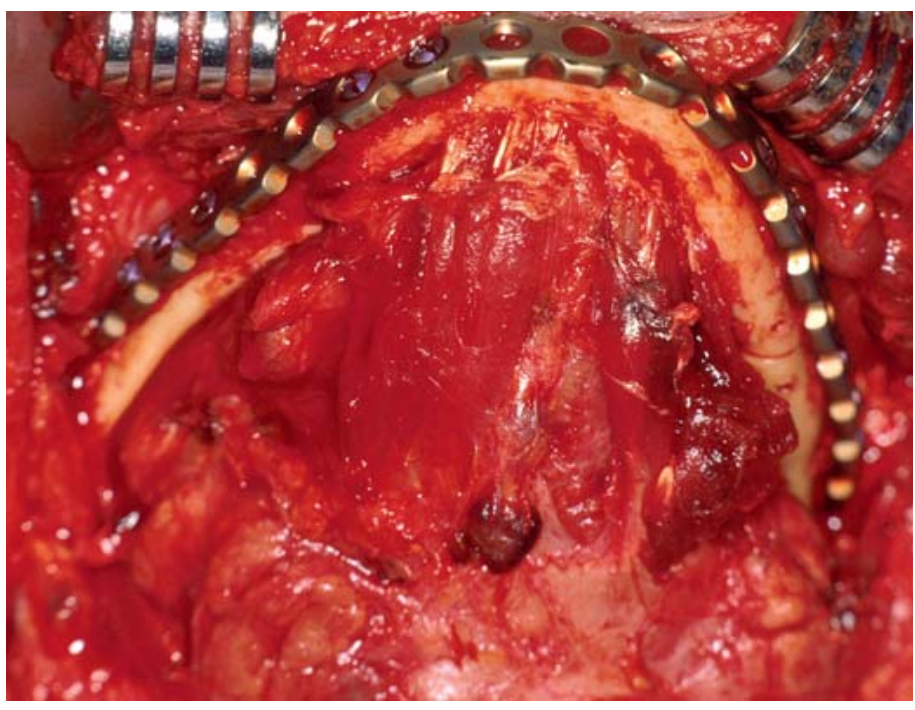

- Abb. 17 Load-bearing-Osteosynthese eines hoch atrophen Unterkiefers über einen extraoralen Zugang.

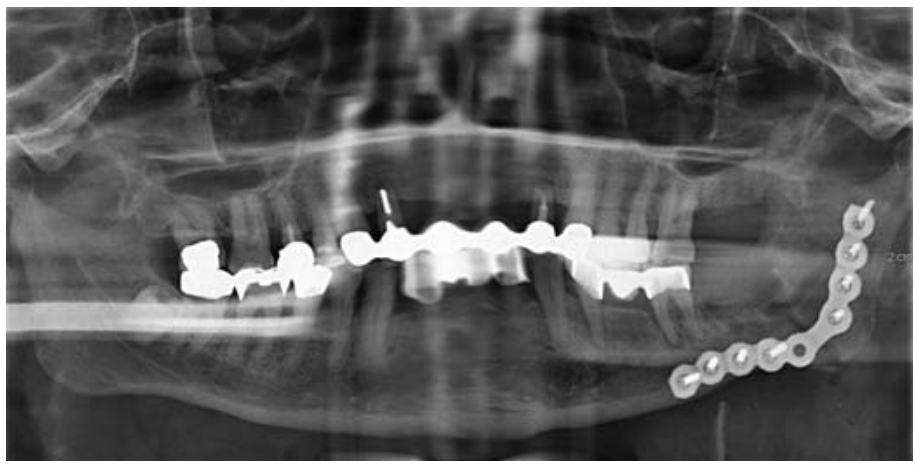

Abb. 18 Load-bearing-Osteosynthese einer Defektfraktur im linken Kieferwinkel. 


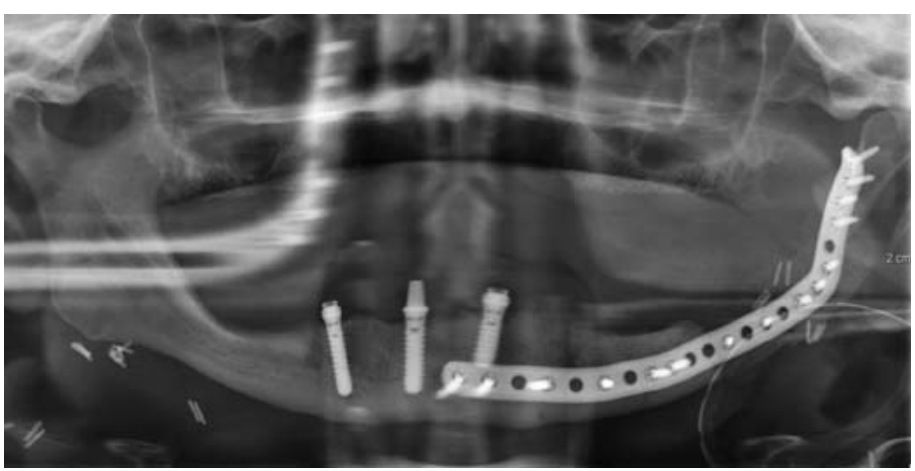

- Abb. 19 Alloplastische Unterkieferrekonstruktion mit CAD/CAMOsteosyntheseplatte (DePuy/Synthes, Schweiz) nach ablativer Tumorchirurgie.

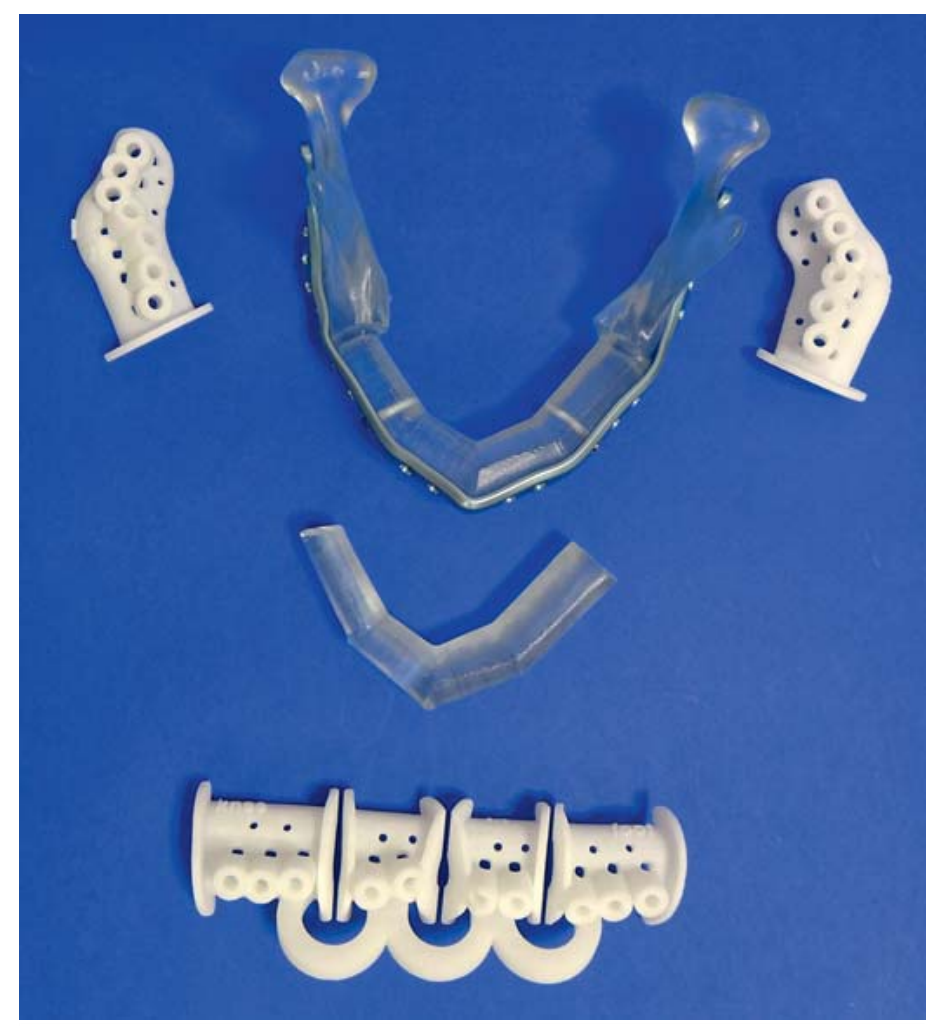

- Abb. 20 Modelle, Operationsschablonen und CAD/CAM-Unterkieferrekonstruktionsplatte zur knöchernen Unterkieferrekonstruktion mittels Fibulatransplantat (Materialise, Belgien, und DePuy/Synthes, Schweiz).

mittlerweile patientenspezifische CAD/CAM-Platten in Kombination mit Operationsschablonen für die Osteotomie des Unterkiefers und des Knochentransplantats etabliert ( $\triangleright$ Abb. 20).

Unter Nichtberücksichtigung der Kosten für das Osteosynthesematerial, liegt der Vorteil einer Rekonstruktionsplatte im Vergleich zu Miniplatten darin, dass bei einem Verlust des Knochentransplantats die Kontinuität des Unterkiefers weiter erhalten bleibt. Der Nachtteil ist darin zu sehen, dass die Gefahr einer postoperativen Plattenexposition nach intra- oder extraoral im Vergleich zu Miniplatte erhöht erscheint.

\section{Prinzipien der Osteosynthese im Mittelgesicht}

Neben der Wiederherstellung der Okklusion steht die anatomisch korrekte Reposition im Fokus bei der Versorgung von traumatischen Verletzungen im Mittelgesicht. Sowohl die knöcherne Höhe als auch die Weite und die Projektion sollte hierbei wiederhergestellt werden. Jede Über- oder Unterkonturierung der Mittelgesichtsknochen kann zu einer ästhetischen Beeinträchtigung führen. Des Weiteren sind funktionelle Probleme wie z. B. eine Okklusionsstörung oder Komplikationen im Bereich der Orbita, wie Diplopie oder ein Enophthalmus, möglich [8].

Auch im Bereich des Mittelgesichts ist die Biomechanik bei der Platzierung der Osteosyntheseplatten bzw. der Rekonstruktion zu beachten [8]. Eine erfolgreiche Rekonstruktion der Mittelgesichtsfrakturen wird dabei durch die Wiederherstellung der sogenannten lateralen und medialen Mittelgesichtspfeiler erreicht ( $\bullet$ Abb. 21). Diese sind dafür verantwortlich, die auftretenden Kaukräfte nach kranial in den Schädel weiterzuleiten. Weiter findet man in diesen Bereichen ein ausreichendes Knochenangebot, um die Platten mittels Osteosyntheseschrauben suffizient zu verankern. Dabei kann in Abhängigkeit vom Frakturmuster die Rekonstruktion von lateral nach medial und/oder von kranial nach kaudal erfolgen [8].

Die typischen Lokalisationen für die Positionierung des Osteosynthesematerials sind im Bereich des lateralen Mittelgesichtspfeilers die Crista zygomaticoalveolaris sowie die Sutura frontozygomatica und im Bereich des medialen Mittelgesichtspfeilers die Regio paranasalis. Bei komplexeren Fällen mit z. B. ausgesprengten Fragmenten im Bereich des Infraorbitalrands kann es notwendig werden, auch hier eine Osteosyntheseplatte zu platzieren. Bei zusätzlichen Frakturen des Jochbogens, des nasoethmoidalen Komplexes, des Oberkiefers und des Alveolarfortsatzes sind auch dort weitere Osteosyntheseplatten möglicherweise notwendig ( $\triangleright$ Abb. 22) [9]. Kleinere Fragmente außerhalb der Mittelgesichtspfeiler erfordern grundsätzlich nicht immer eine osteosynthetische Fixierung. Hier reicht es häufig aus, diese locker zu reponieren und sie ggf. z. B. mit Fibrinkleber zu stabilisieren. Größere Fragmente, z.B. im Bereich der Kiefer- und Stirnhöhlenvorderwand, sollten mit dünnem Osteosynthesematerial fixiert werden.

Die Osteosynthese im Bereich des Mittelgesichts ist ausschließlich die Domäne der Miniplatten, wobei auch hier die modernen Osteosynthesesysteme multimodal aufgebaut sind, sodass mit einem System verschiedene Plat- 


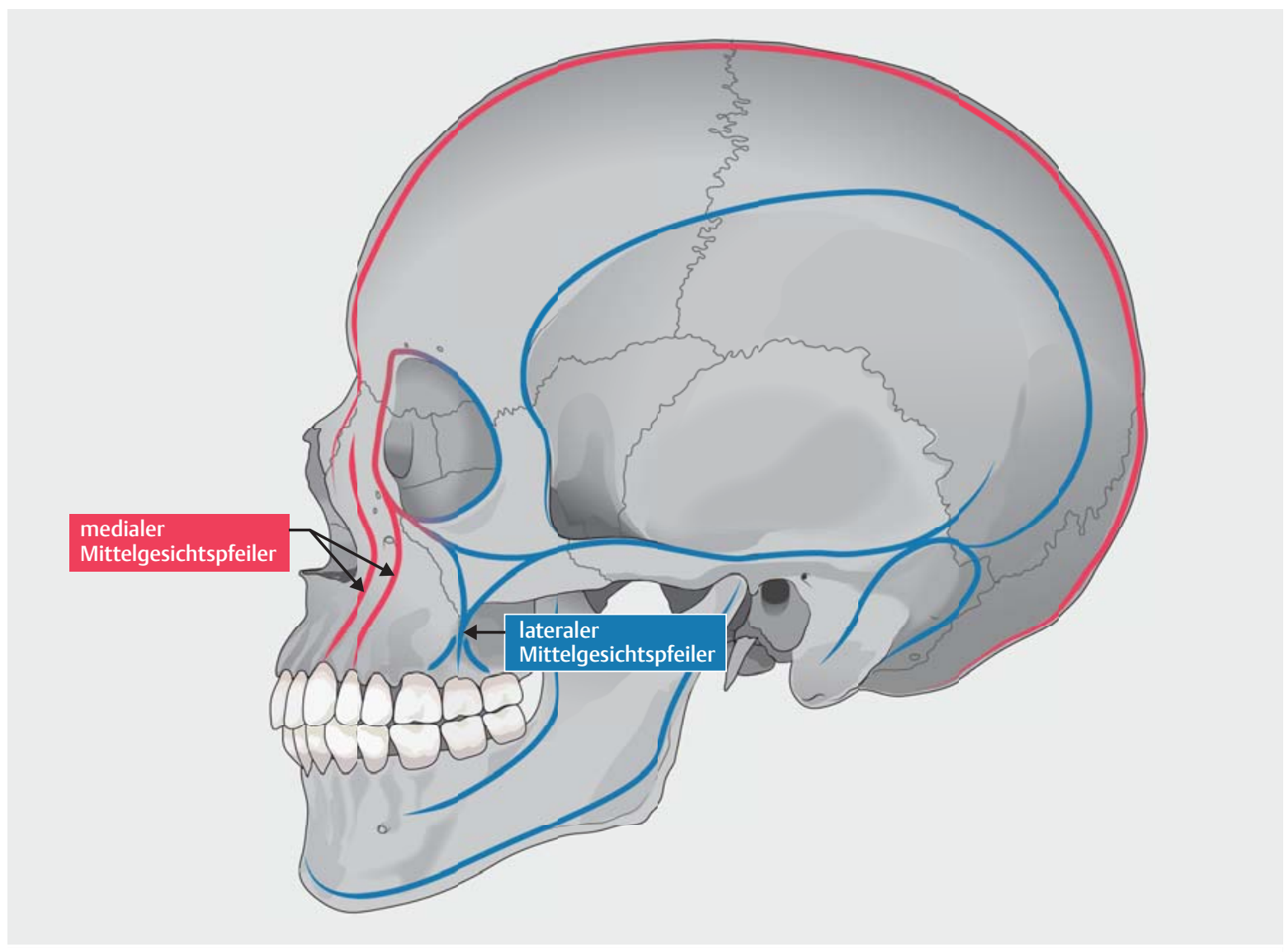

- Abb. 21 Schematische Darstellung der Kraftvektoren (Trajektorien) des Mittelgesichts.

tenstärken mit ein und derselben Schraube verwendet werden können ( $\bullet$ Abb. 1 b). Die durch das Osteosynthesematerial aufzunehmenden Kräfte sind nicht vergleichbar mit denen im Unterkiefer. Deshalb sind, nach den Erfahrungen in der Autorenklinik, eine Plattenprofilstärke von $0,5 \mathrm{~mm}$ ausreichend. Nur beim Vorhandensein z.B. größerer Knochenlücken insbesondere im Bereich des lateralen Mittelgesichtspfeilers ist eine Plattenprofilstärke von 0,7 oder $0,8 \mathrm{~mm}$ zu empfehlen. Im Bereich des Infraorbitalrandes und zur Fixierung einzelner Knochenfragmente z.B. im Bereich der Kiefer- oder Stirnhöhlenvorderwand ist sogar noch dünneres Osteosynthesematerial (z. B. 0,4 mm Profilstärke) als suffizient zu erachten.

Auch hier konzentrieren sich die neuesten Entwicklungen inzwischen auf anatomisch präformierte Osteosyntheseplatten ( $\triangleright$ Abb. 23). Wie im Unterkiefer, soll dies auch im Mittelgesicht das Konturieren der Platten erleichtern und die Operationszeiten reduzieren.

Hinsichtlich der Schrauben sind Durchmesser zwischen 1,3 und 1,5 mm und Längen zwischen 4-6 mm zu empfehlen. Winkelstabile Schrauben kommen bis dato im Bereich des Mittelgesichts i.d.R. noch nicht zum Einsatz.

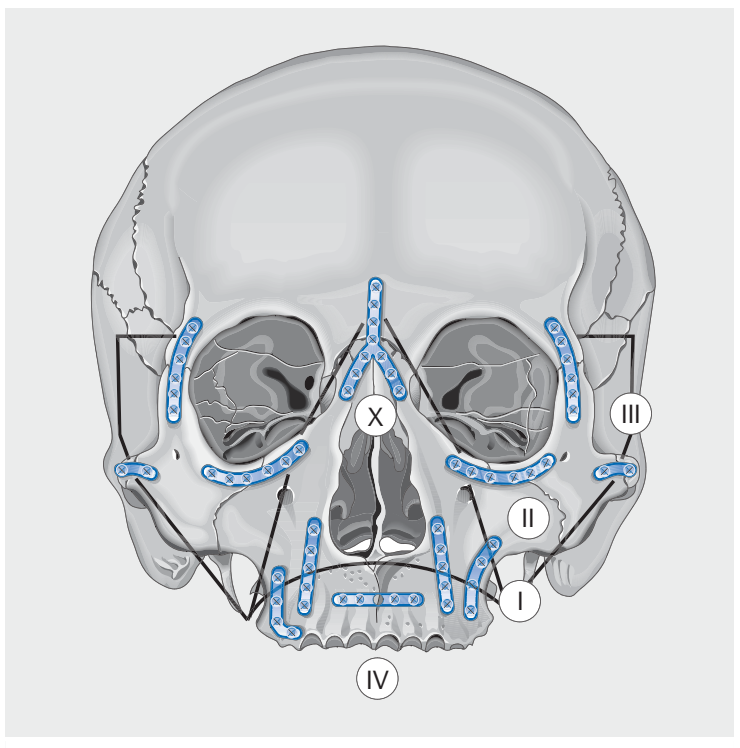

- Abb. 22 Zentrale Mittelgesichtsfrakturen. I: LeFort-IFraktur, II: LeFort-II-Fraktur, III: LeFort-III-Fraktur, IV: Sagittalfraktur im Bereich des Oberkiefers, X: Nasenbeinfrakturen. blau: potenzielle Plattenlokalisationen. Quelle: Hell B. Zentrale Mittelgesichtsfrakturen. In: Wirth C, Mutschler W, Kohn D et al., Hrsg. Praxis der Orthopädie und Unfallchirurgie. 3. Aufl. Stuttgart: Thieme; 2013. 

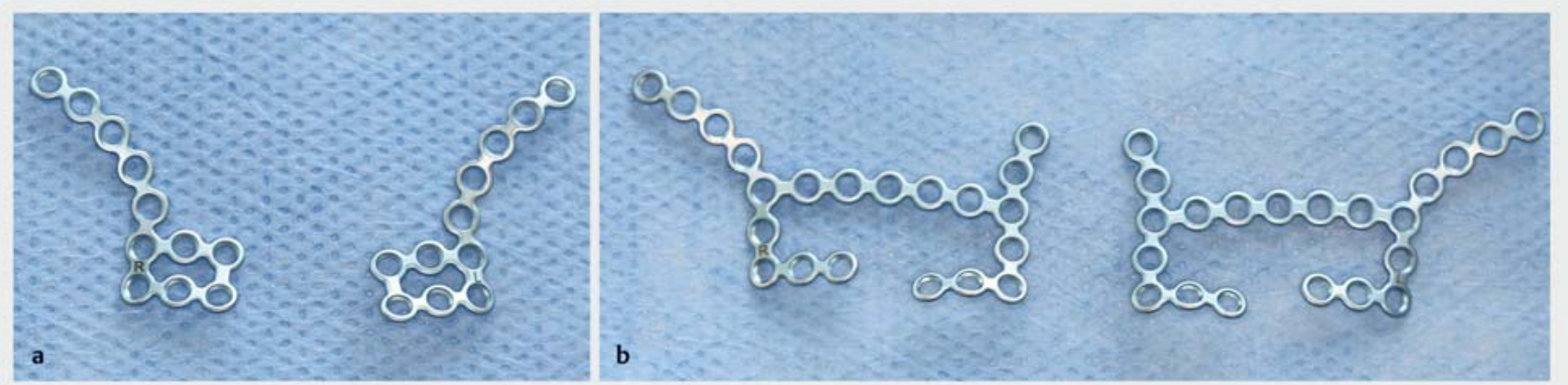

- Abb. 23 Anatomisch präformierte Platten für das Mittelgesicht. a Isoliert für lateralen Mittelgesichtspfeiler. b Für lateralen und medialen Mittelgesichtspfeiler in Kombination (KLS-Martin, Deutschland).

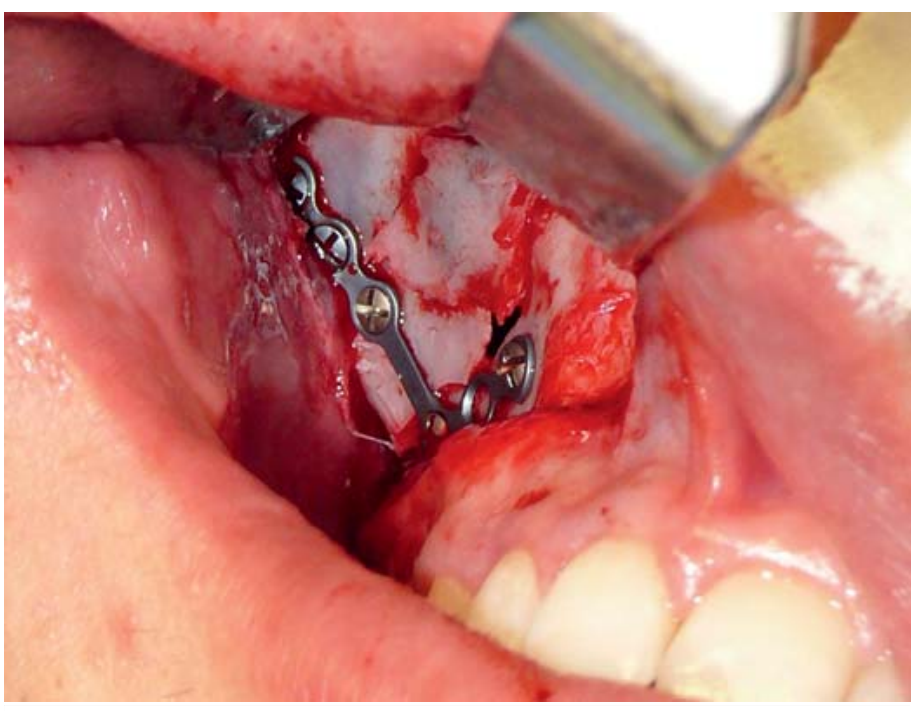

- Abb. 24 Reposition einer unkomplizierten Fraktur des Jochbeinkomplexes und Osteosynthese mit einer Platte im Bereich der Crista zygomaticoalveolaris.

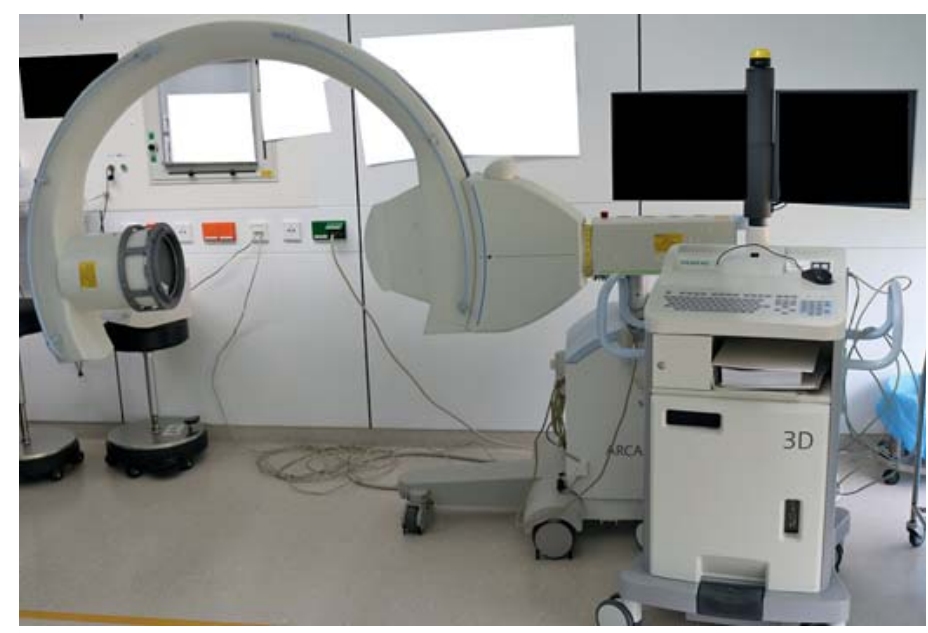

- Abb. 25 3-D-C-Bogen zur intraoperativen Anwendung (Siemens, Deutschland).

\section{Anwendungsbeispiele der \\ Osteosynthese im Mittelgesicht}

\section{Frakturen des Jochbeinkomplexes}

Bei unkomplizierten Frakturen des Jochbeinkomplexes ist es häufig ausreichend, über einen intraoralen Zugang nach Reposition eine einzige Platte im Bereich der Crista zygomaticoalveolaris zu fixieren ( $\bullet$ Abb. 24). Hiermit kann eine ausreichende Stabilität erreicht werden. Bei dieser Art der Versorgung ist eine intraoperative 3-D-Bildgebung z.B. unter Einsatz eines 3-D-C-Bogens ( $\triangleright$ Abb. 25) oder eines intraoperativen DVT sinnvoll, um neben der osteosynthetisch stabilisierten Fraktur auch die Reposition im Bereich der übrigen Frakturlinien des Jochbeinkomplexes, wie die Sutura frontozygomatica und den Infraorbitalrand, zu visualisieren $[10,11]$. Alternativ ist hier natürlich auch eine Palpation möglich. Im Bereich des Infraorbitalrandes ist dies meist suffizient möglich. Im Bereich des lateralen Orbitarandes bzw. der Sutura frontozygomatica sind jedoch Fehlinterpretationen möglich. Sollte eine ausreichende Stabilisierung des Jochbeinkomplexes über eine Platte im Bereich der Crista zygomaticoalveolaris nicht möglich sein, werden weitere Osteosyntheseplatten, z. B. im Bereich der Sutura frontozygomatica oder ggf. auch im Bereich des Infraorbitalrandes, notwendig.

Während für die Osteosynthese im Bereich der Crista zygomaticoalveolaris ein intraoraler Zugang über einen vestibulären Schnitt ausreichend ist, empfiehlt sich als operativer Zugangsweg zum lateralen Orbitarand der sogenannte Blepharoplastikzugang im Bereich des lateralen Augenlids [4]. Hierdurch können sichtbare Narben in der Regel vermieden werden. Bei einer zusätzlich notwendigen Osteosyntheseplatte im Bereich des Infraorbitalrandes, kann diese nicht nur durch einen zusätzlichen Zugang durch das Unterlid eingebracht werden. Hierzu ist in vielen Fällen auch die Verwendung des bereits vorhandenen intraoralen Zugangs möglich, da die Osteosynthese- 
platte auch kaudal des N. infraorbitalis zur Stabilisierung des Jochbeinkomplexes fixiert werden kann. Nach Autorenmeinung wird somit ein zusätzlicher Zugang zum Infraorbitalrand durch das Unterlid nur bei ausgesprengten Fragmenten, komplexen Verlagerungen sowie bei der Indikation zur Orbitarekonstruktion notwendig.

\section{Frakturen des Jochbein-Orbita-Komplexes}

Häufig zeigt sich bei Frakturen des Jochbeinkomplexes auch eine Dislokation im Bereich des Orbitabodens. Hierbei stellt sich regelhaft die Frage, ob der Orbitaboden einer operativen Rekonstruktion zusätzlich zur Reposition und Stabilisation des Jochbeinkomplexes bedarf. Die Erfahrung hat hier gezeigt, dass häufig eine alleinige Reposition und Fixierung des Jochbeinkomplexes nach den oben beschriebenen Prinzipien ausreichend ist, um auch den Orbitaboden suffizient und anatomisch regelrecht zu reponieren. Eine Orbitarekonstruktion ist in solchen Fällen nur selten notwendig. Zur intraoperativen Entscheidungshilfe hierüber ist die bereits erwähnte intraoperative 3-D-C-Bogen- oder DVT-Bildgebung zu empfehlen. Diese erlaubt die intraoperative Beurteilung der Reposition und ermöglicht somit die Entscheidung, ob zusätzlich eine Orbitarekonstruktion indiziert ist ( $\bullet$ Abb. 26) $[10,11]$.

\section{Wenig komplexe zentrolaterale Mittel- gesichtsfrakturen}

Bei wenig komplexen zentrolateralen Mittelgesichtsfrakturen müssen neben den Frakturen im Bereich des Jochbein- oder Jochbein-Orbita-Komplexes auch die Frakturen im zentralen Mittelgesicht adressiert werden. Hierzu sollte das zusätzliche Osteosynthesematerial im Bereich der paranasalen bzw. zentralen Mittelgesichtspfeiler fixiert werden ( $\triangleright$ Abb. 27 ).

\section{LeFort-Frakturen}

Bei den LeFort-Frakturen ist entweder der gesamte Oberkiefer (LeFort I), die Gesichtspyramide (LeFort II) oder das gesamte Mittelgesicht (LeFort III) von der Schädelbasis abgesprengt ( $\mathbf{A} \mathbf{b} \mathbf{b}$. 22). Die Osteosynthese erfolgt analog der beschriebenen Prinzipien entlang der zentralen und lateralen Mittelgesichtspfeiler mittels Miniplattenosteosynthese. Sollte das Mittelgesicht jedoch eingestaucht sein und daraus eine fehlerhafte Okklusion resultieren, wird ggf. eine komplette oder zusätzliche LeFort-IOsteotomie mit Down Fracture des Oberkiefers wie bei einer Dysgnathieoperation notwendig, um die Okklusion regelgerecht einzustellen. Dies betrifft gleichermaßen LeFort-I-, -II und -III-Frakturen.

\section{Merke}

Eine intraoperative 3-D-Bildgebung ermöglicht die Visualisierung der Reposition bereits während des Eingriffs. Dies ermöglicht minimalinvasive Zugänge und es werden Revisionsoperationen verhindert.

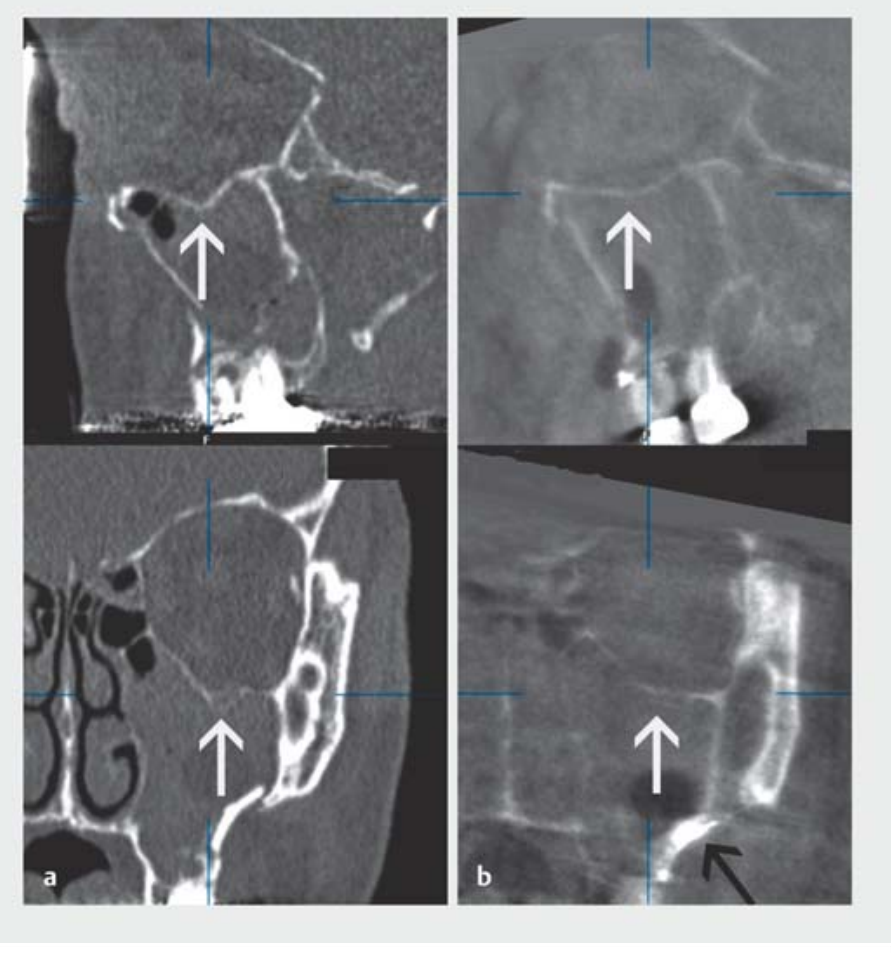

Abb. 26 Isoliert von intraoral versorgte Fraktur des Jochbein-OrbitaKomplexes. a Präoperativer CT-Befund mit Dislokation des Orbitabodens. b Intraoperative 3-D-C-Bogen-Kontrolle. Durch alleinige Reposition des Jochbein-Orbita-Komplexes kam es zu einer suffizienten Reposition des Orbitabodens. Eine Orbitarekonstruktion war nicht notwendig.

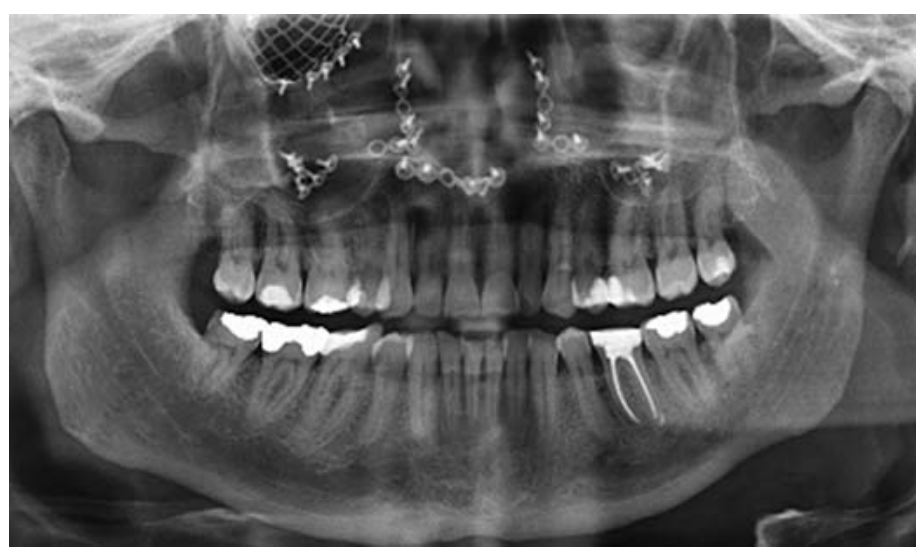

- Abb. 27 Mittels Miniplattenosteosynthese versorgte zentrolaterale Mittelgesichtsfraktur. In die rechte Orbita wurde ein anatomisch präformiertes Implantat implantiert.

\section{Orbitawandfrakturen}

Orbitawandfrakturen können einerseits isoliert, andererseits auch in Kombination mit zentralen, lateralen bzw. zentrolateralen Mittelgesichtsfrakturen auftreten. Dabei sollten prinzipiell vor einer Orbitarekonstruktion immer erst die Mittelgesichtsfrakturen anatomisch regelgerecht versorgt werden. 


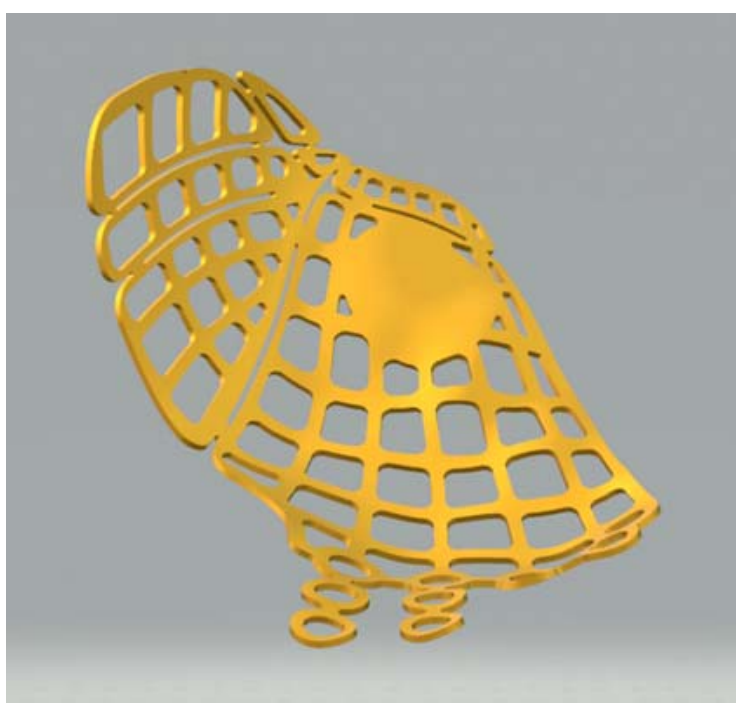

- Abb. 28 3-D-Modell eines anatomisch präformierten Orbitagitters (DePuy/Synthes, Schweiz).

Als operativer Zugang für die Orbitarekonstruktion empfiehlt sich laut Autorenmeinung ein transkonjunktivaler Zugang, der ggf. transkarunkulär erweitert werden kann [4]. Hierdurch besteht die Möglichkeit der Exploration der Orbita bis zu $270^{\circ}$, da über diesen Zugang auch die mediale Orbitawand bis zur Schädelbasis disseziert werden kann. Eine schonende Präparation ist dabei von größter Wichtigkeit, um einerseits den $\mathrm{N}$. infraorbitalis nicht zusätzlich zu schädigen und andererseits die feinen Augenmuskeln nicht zu verletzen.

Zur Orbitawandrekonstruktion werden heutzutage fast einheitlich Titangitter empfohlen. Von Autorenseite können Akutversorgung insbesondere anatomisch präformierte Orbitarekonstruktionsgitter empfohlen werden, die bereits die typische Form des Orbitabodens vorgeben ( $\vee$ Abb. 28). Von entscheidender Bedeutung ist die Positionierung des Titangitters im Bereich der posterioren Auflage, die im Rahmen der Orbitadissektion aufgesucht werden muss. Weiter muss streng darauf geachtet werden, dass die implantierten Gitter posterior nicht nach kranial abstehen oder periorbitales Gewebe einklemmen und so möglicherweise die Augenmuskeln in ihrer Funktion komprimitieren.

Eine deutliche Fehlpositionierung des Gitters kann dabei durch den Einsatz einer intraoperativen 3-D-Bildgebung mit hoher Sicherheit verhindert werden [11].

Neben der in der Autorenklinik bei Orbitarekonstruktionen obligaten intraoperativen 3-D-Bildgebung empfiehlt sich weiterhin bei komplexen Fällen die Verwendung der intraoperativen Instrumentennavigation. Durch die zunehmende Tendenz hin zu patientenspezifischen Implantaten, die präoperativ computerassistiert geplant und mittels CAD-Verfahren additiv mittels selektiv laser melting hergestellt werden, tritt diese jedoch heutzutage mehr und mehr in den Hintergrund. Die Passung solcher patientenspezifischer Implantate erreicht mittlerweile eine solch hohe Präzision, dass eine relevante Fehlpositionierung und mehrfache Korrektur der Gitterlage, die mittels der Navigation strahlungsfrei durchgeführt werden kann, nicht mehr erforderlich ist ( $\mathbf{A b b}$. 29) [12].

\section{Merke}

Bei Orbitawandrekonstruktionen sind Titangitter der Rekonstruktionsmaterial der Wahl. Anatomisch präformierte oder patientenspezifische Implantate erleichtern dabei die Rekonstruktion erheblich. Die Fehlpositionierung des Orbitaimplantats kann durch den Einsatz einer intraoperativen 3-D-Bildgebung mit hoher Sicherheit verhindert werden.

\section{Komplexe Mittelgesichtsfrakturen}

Bei komplexen Mittelgesichtsfrakturen muss im Bedarfsfall neben einem intraoralen und transkonjunktivalen Zugang noch ein koronarer Zugang gewählt werden, um ggf. Jochbögen, Supraorbitalrand sowie Stirnhöhle einschließlich des nasoethmoidalen Komplexes osteosynthetisch versorgen zu können. In Abhängigkeit vom Frakturmuster, empfiehlt sich die Mittelgesichtspfeilerrekonstruktion von lateral nach medial und/oder von kranial nach kaudal. Weiter sollte immer von weniger komplex nach komplex rekonstruiert werden. Demnach ist es oft hilfreich, die Fraktur durch eine anatomische Reposition und Fixierung von größeren Komplexen zu vereinfachen, bevor man die Mittelgesichtspfeiler und die Okklusion wiederherstellt.

Bei größeren Knochenverlusten insbesondere im Bereich der Kieferhöhlenvorderwand oder der Stirnhöhlenvorderwand empfiehlt es sich, diese Strukturen z. B. mittels Titangittern zu rekonstruieren ( $\triangleright$ Abb. 30 ).

Bei zusätzlichen Frakturen im Bereich der Maxilla im Sinne von Sagittalfrakturen sollte eine stabilisierende Osteosyntheseplatte transversal unterhalb der Apertura piriformis platziert werden. Um ein Auseinanderweichen im Bereich des posterioren Gaumens zu vermeiden und eine zusätzliche Stabilisierung zu erreichen, empfiehlt sich nach Autorenmeinung die Platzierung einer Osteosyntheseplatte im Bereich des Gaumens. Eine solche kann bei modernen Osteosynthesesystemen mit winkelstabilen Schrauben transgingival im Sinne eines Fixateur externe unkompliziert erfolgen ( $\bullet$ Abb. 31$)$. Hierdurch kann z.B. auf einen transpalatinalen Draht verzichtet werden. Zur passiven regelgerechten Einstellung der Okklusion wird ggf. wie bereits im Abschnitt LeFort-Frakturen beschrieben, eine komplette oder zusätzliche LeFort-I-Osteotomie mit Down Fracture des Oberkiefers notwendig. 

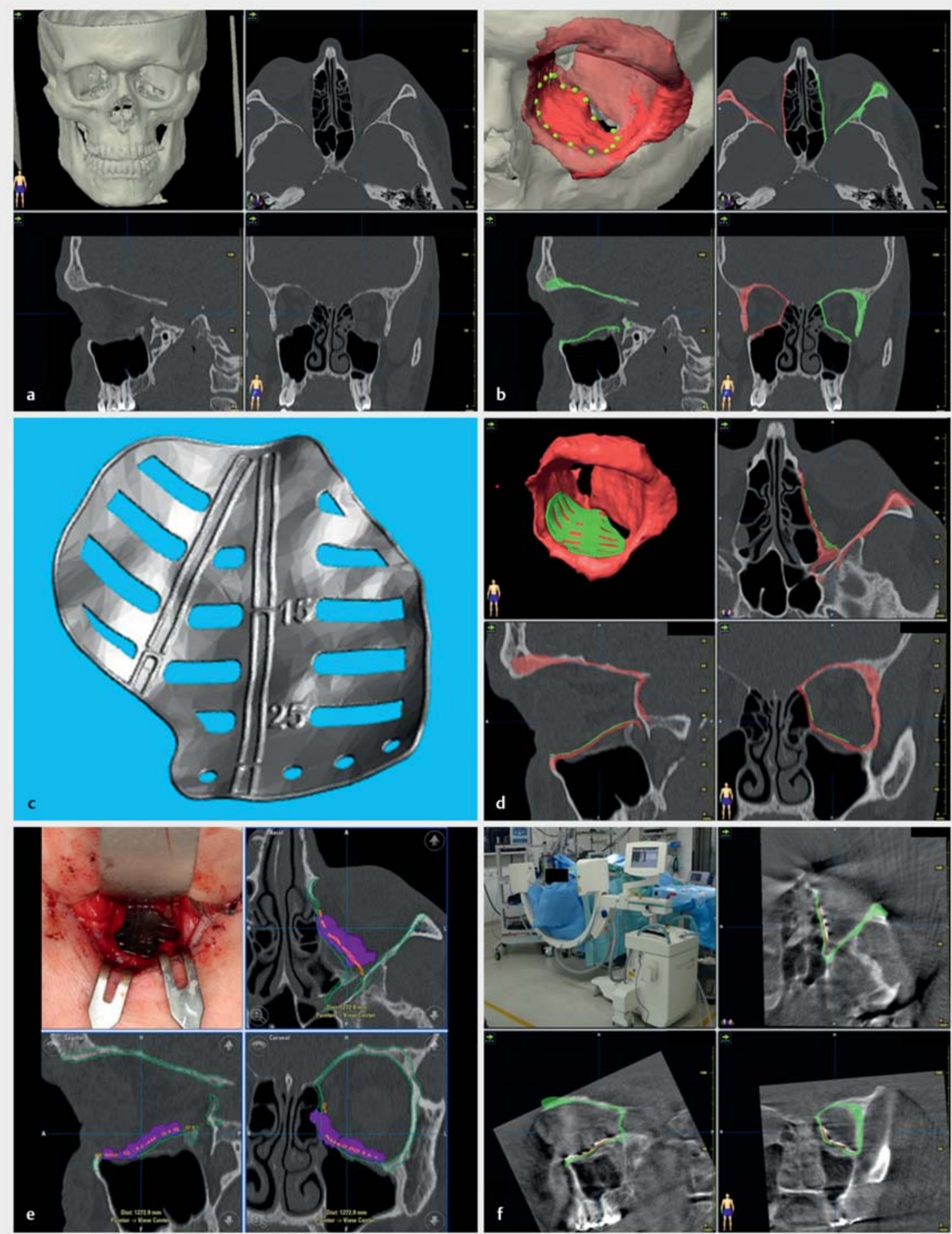

- Abb. 29 Computerassistierte Sekundärrekonstruktion der linken Orbita mittels eines patientenspezifischen Implantats. a Insuffizient rekonstruierte linke Orbita. b Virtuelle Operationsplanung mittels iPlanCMF 3.0 (Brainlab, Deutschland). c Computer-Aided Design des patientenspezifischen Implantats (KLS-Martin, Deutschland). d Simulation der Rekonstruktion mit virtuell implantiertem patientenspezifischen Implantat. e Implantation des patientenspezifischen Implantats über transkonjunktivalen Zugang und Überprüfung der Position mittel Navigationssystem (Brainlab, Deutschland). Dabei spiegelt die lila Kontur die Oberfläche des Implantats im Situs wider. $f$ Multiplanare Ansicht der intraoperativen 3-D-C-Bogen-Bildgebung nach Fusion mit der präoperativen Planung. Das Implantat kommt exakt in der geplanten Position zu liegen. 


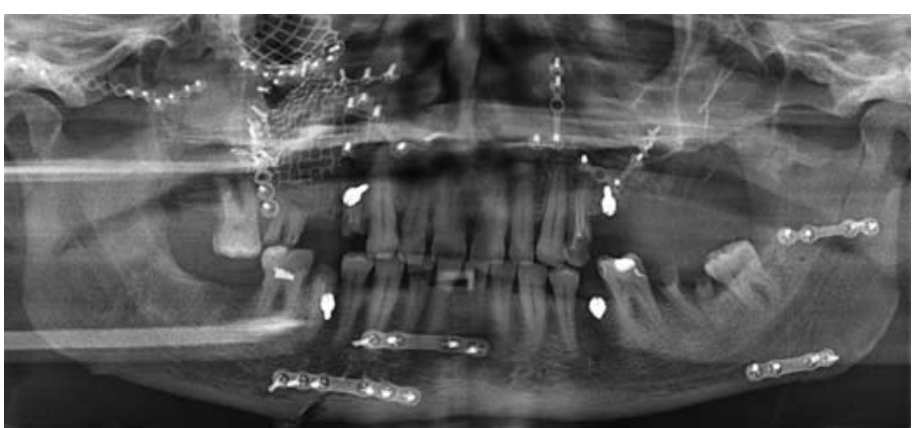

- Abb. 30 Mittels Miniplattenosteosynthese versorgte panfaziale Fraktur. Im Bereich der rechten Kieferhöhlenvorderwand wurde ein Titangitter zur Rekonstruktion inseriert.

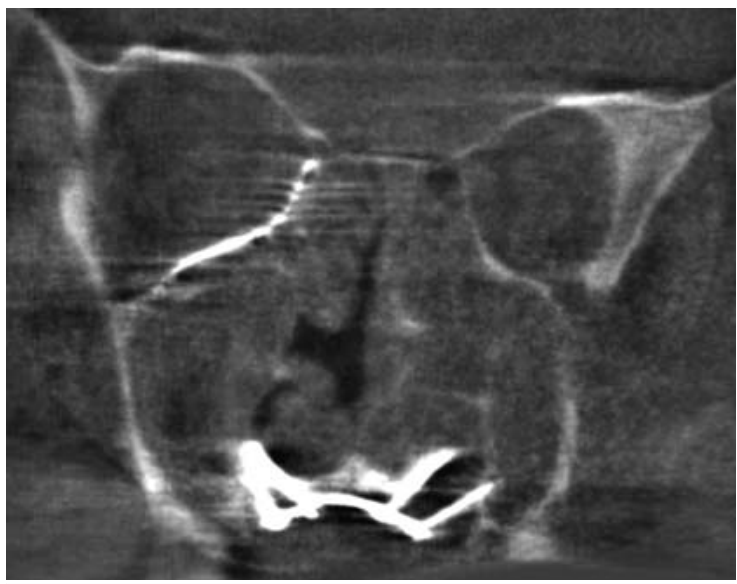

- Abb. 31 Transgingival eingebrachte winkelstabile Osteosyntheseplatte im Bereich des Gaumens im Sinne eines Fixateur externe. Hierdurch wurde eine Oberkiefersagittalfraktur im posterioren Bereich stabilisiert. Auf einen transpalatinalen Draht konnte somit verzichtet werden. In die rechte Orbita wurde ein anatomisch präformiertes Implantat implantiert.

Insbesondere bei solch komplexen Frakturmustern empfiehlt sich der Einsatz der computerassistierten Chirurgie mit präoperativer Planung, patientenspezifischen Implantaten, intraoperativer Instrumentennavigation sowie intraoperativer 3-D-Bildgebung [10-12].

\section{Panfaziale Frakturen}

Bei panfazialen Frakturen mit Frakturen im Bereich des Ober- und Unterkiefers ( $\boldsymbol{A} \mathbf{A b b} \mathbf{3} \mathbf{3 0}$ ) sollte hinsichtlich der Sequenzierung des operativen Vorgehens primär geklärt werden, ob man von kranial nach kaudal oder ggf. von kaudal nach kranial rekonstruiert. Im Vordergrund steht auch hier wie bei allen Rekonstruktionen immer die Wiederherstellung der Okklusion sowie der knöchernen korrekten Anatomie hinsichtlich Breite, Höhe und anterior-posteriorer Projektion. Dabei empfiehlt sich grundsätzlich, von den weniger komplexen Frakturen zu den komplexeren zu rekonstruieren.

\section{FAZIT}

- Bei der Osteosynthese im Bereich des Gesichtsschädels spielt neben der Anatomie die Biomechanik die entscheidende Rolle hinsichtlich der Positionierung des Osteosynthesematerials.

- Bei Unterkieferfrakturen mit guter Anlagerungsfläche der Frakturfragmente ist die Miniplattenosteosynthese mit monokorticalen Schrauben im Sinne einer Load-sharing-Osteosynthese das Mittel der Wahl. Das Prinzip der Load-bearingOsteosynthese unter Einsatz von dickerem Osteosynthesematerial in Kombination mit bikortikalen Schrauben sollte eingesetzt werden, wenn keine ausreichende Anlagerungsfläche des Knochens im Bruchspaltbereich möglich ist.

- Im Mittelgesicht kommen ausschließlich Miniplatten zum Einsatz. Diese sollten primär im Bereich der medialen und lateralen Mittelgesichtspfeiler implantiert werden. In Abhängigkeit vom Frakturmuster sollte die Rekonstruktion von lateral nach medial und/oder von kranial nach kaudal erfolgen.

- Auf extraorale Zugänge sollte, wenn immer möglich, sowohl im Unterkiefer als auch im Bereich des Mittelgesichts verzichtet werden. Sollten diese dennoch notwendig sein, werden die Zugänge so gewählt werden, dass sie möglichst unsichtbar bleiben.

- Moderne Osteosynthesesysteme sind so konzeptioniert, dass alle Platten mit allen Schrauben verwendet werden können. Im Bereich der Unterkiefersysteme können zusätzlich sowohl winkelstabile als auch nicht winkelstabile Schrauben miteinander kombiniert werden.

- Die neuesten Entwicklungen gehen in Richtung anatomisch präformierter Osteosyntheseplatten. Diese sollen das Konturieren der Platten erleichtern, die Plattenfrakturgefahr mindern und Operationszeiten reduzieren.

- Die computerassistierte Chirurgie ist mittlerweile ein integraler Bestandteil der modernen Gesichtsschädelrekonstruktion. Neben der präoperativen Computerplanung bietet insbesondere der Einsatz einer intraoperativen 3-D-Bildgebung große Vorteile.

- Patientenspezifische mittels CAD-CAM-Verfahren hergestellte Implantate finden in der Akuttraumatologie derzeit noch wenig Anwendung. Die Anwendung solcher patientenspezifischer Implantate konzentriert sich auf Sekundärrekonstruktionen oder die Rekonstruktion von größeren Defekten nach ablativer Chirurgie. 


\section{Interessenkonflikt}

Referententätigkeit für Firmen DePuy/Synthes und KLS Martin.

Autorinnen/Autoren

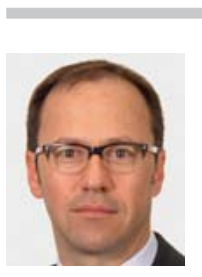

\section{Frank Wilde}

Priv.-Doz. Dr. med. Dr. med. dent., Leitender Oberarzt und stellvertretender ärztlicher Direktor der Klinik für Mund-, Kiefer- und plastische Gesichtschirurgie am Bundeswehrkrankenhaus

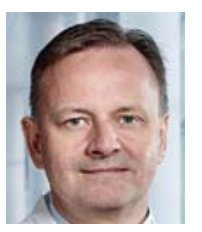

\section{Alexander Schramm}

Prof. Dr. med. Dr. med. dent. habil., Ärztlicher Direktor der Klinik für Mund-, Kiefer- und Gesichtschirurgie am Universitätsklinikum Ulm

Korrespondenzadresse

Priv.-Doz. Dr. Dr. Frank Wilde

Leitender Oberarzt und stellv. ärztl. Direktor Klinik für Mund-, Kiefer- und plastische Gesichtschirurgie Bundeswehrkrankenhaus Ulm

Oberer Eselsberg 40

89075 Ulm

Oberarzt der Klinik für Mund-, Kiefer- und Gesichtschirurgie Universitätsklinikum Ulm

Albert-Einstein-Allee 11

89075 Ulm

frank.wilde@uni-ulm.de

\section{Literatur}

[1] Rüedi T, Buckley R, Moran C. AO Philosophy and Evolution. Im Internet: https://www2.aofoundation.org/wps/portal/surgery? showPage $=$ diagnosis\&bone $=$ CMF\&segment $=$ Over view; Stand: 27.08.2018

[2] Cienfuegos R, Cornelius CP, Ellis E, Kushner G. AOCMF Surgery Reference Mandible. Figari M, Sánchez Aniceto G, Buchbinder D, eds. https://www2.aofoundation.org/wps/portal/surgery? showPage $=$ diagnosis\&bone $=C M F \&$ segment $=$ Mandible; Stand: 27.08.2018
[3] Champy M, Loddé JP, Schmitt R et al. Mandibular osteosynthesis by miniature screwed plates via a buccal approach. J Maxillofac Surg 1978; 6: 14-21

[4] Ellis E, Zide M. Surgical Approaches to the facial Skeleton. 2nd ed. Philadelphia: Lippincott Williams \& Wilkins; 2006

[5] Cornelius C-P, Probst F, Rathgeber J-P et al. Zur Osteosynthese bei Frakturen im zahnlosen atrophen Unterkiefer - Möglichkeiten zum Hardware Downsizing, Lingual Plating. OP-JOURNAL 2013; 29: 164-174 doi:10.1055/s-0033-1350819

[6] Wilde F, Cornelius C-P, Schramm A. Computer-assisted mandibular reconstruction using a patient-specific reconstruction plate fabricated with computer-aided design and manufacturing techniques. Craniomaxillofac Trauma Reconstr 2014; 7: 158-166

[7] Wilde F, Hanken H, Probst F et al. Multicentre Study on the use of patient-specific CAD/CAM reconstruction plates for mandibular reconstructions. Int J Comput Assist Radiol Surg 2015; 10: 2035-2051

[8] Cornelius C-P, Gellrich N, Hillerup S, Kusumoto K, Schubert W. AOCMF Surgery Reference Midface. Ellis E, Kazuo Shimozato K, Buchbinder D, eds. https://www2.aofoundation.org/wps/portal $/$ surgery? showPage $=$ diagnosis\&bone $=$ CMF\&segment = Midface; Stand: 27.08.2018

[9] Hell B. Zentrale Mittelgesichtsfrakturen. In: Wirth C, Mutschler W, Kohn D, Pohlemann T, Hrsg. Praxis der Orthopädie und Unfallchirurgie. 3., vollständig überarbeitete Auflage. Stuttgart: Thieme; 2013. doi:10.1055/b-002-23558

[10] Wilde F, Lorenz K, Ebner AK et al. Intraoperative imaging with a 3D C-arm system after zygomatico-orbital complex fracture reduction. J Oral Maxillofac Surg 2013; 71: 894-910

[11] Wilde F, Schramm A. Intraoperative Imaging in Orbital and Midface Reconstruction. Facial Plast Surg 2014; 30: 545-553

[12] Wilde F, Schramm A. [Computer-aided reconstruction of the facial skeleton: Planning and implementation in clinical routine]. HNO 2016; 64: 641-649

Bibliografie

DOI https://doi.org/10.1055/a-0724-6264

Online-publiziert 30.11.2018 | OP-JOURNAL 2019; 35: 45-61

(c) Georg Thieme Verlag KG Stuttgart · New York

ISSN 0178-1715 